IZA DP No. 10090

Inequality and Growth:

The Role of Human Capital with Heterogeneous Skills

Kirill Borissov

Stefano Bosi

Thai Ha-Huy

Leonor Modesto

July 2016 


\title{
Inequality and Growth: The Role of Human Capital with Heterogeneous Skills
}

\author{
Kirill Borissov \\ European University of St. Petersburg \\ Stefano Bosi \\ Université d'Evry \\ Thai Ha-Huy \\ Université d'Evry \\ Leonor Modesto \\ UCP, Católica Lisbon School of Business \& Economics and IZA
}
Discussion Paper No. 10090
July 2016

IZA

P.O. Box 7240

53072 Bonn

Germany

\author{
Phone: $+49-228-3894-0$ \\ Fax: +49-228-3894-180 \\ E-mail: iza@iza.org
}

\begin{abstract}
Any opinions expressed here are those of the author(s) and not those of IZA. Research published in this series may include views on policy, but the institute itself takes no institutional policy positions. The IZA research network is committed to the IZA Guiding Principles of Research Integrity.

The Institute for the Study of Labor (IZA) in Bonn is a local and virtual international research center and a place of communication between science, politics and business. IZA is an independent nonprofit organization supported by Deutsche Post Foundation. The center is associated with the University of Bonn and offers a stimulating research environment through its international network, workshops and conferences, data service, project support, research visits and doctoral program. IZA engages in (i) original and internationally competitive research in all fields of labor economics, (ii) development of policy concepts, and (iii) dissemination of research results and concepts to the interested public.
\end{abstract}

IZA Discussion Papers often represent preliminary work and are circulated to encourage discussion. Citation of such a paper should account for its provisional character. A revised version may be available directly from the author. 


\section{ABSTRACT \\ Inequality and Growth: The Role of Human Capital with Heterogeneous Skills*}

We extend the Lucas' 1988 model introducing two classes of agents with heterogeneous skills, discount factors and initial human capital endowments. We consider two regimes according to the planner's political constraints. In the first regime, that we call meritocracy, the planner faces individual constraints. In the second regime the planner faces an aggregate constraint, redistributing. We find that heterogeneity matters, particularly with redistribution. In the meritocracy regime, the optimal solution coincides with the BGP found by Lucas (1988) for the representative agent's case. In contrast, in the redistribution case, the solution for time devoted to capital accumulation is never interior for both agents. Either the less talented agents do not accumulate human capital or the more skilled agents do not work. Moreover, social welfare under the redistribution regime is always higher than under meritocracy and it is optimal to exploit existing differences. Finally, we find that inequality in human capital distribution increases in time and that, in the long run, inequality always promotes growth.

JEL Classification: J24, O15, O40

Keywords: $\quad$ human capital, heterogeneous skills

Corresponding author:

Leonor Modesto

Católica Lisbon School of Business and Economics

Portuguese Catholic University

Palma de Cima

1649-023 Lisboa

Portugal

E-mail:Irm@ucp.pt

\footnotetext{
${ }^{*}$ We acknowledge the support from FCT — Portuguese Foundation of Science and Technology for the project PTDC/IIM-ECO/4831/2014.
} 


\section{Introduction}

Though the consideration of human capital as a source of economic growth and development goes back at least to Adam Smith (1776), ${ }^{1}$ for a long time the concept of human capital was dormant. Interest in the economic role of human capital was awakened in the late 1950s and early 1960s through the writings of Mincer $(1958,1962)$, Schultz $(1961,1962)$ and Becker (1962, 1964), who rekindled this concept by emphasizing its importance in explaining earnings differentials and its links with economic growth.

Human capital as an engine of growth was incorporated into growth theory by Uzawa (1965). The emergence of a new endogenous growth literature stimulated the interest of economists in the role of human capital as a determinant of economic growth. In his widely cited paper, Lucas (1988) shows that the growth rate of per capita income depends on the growth rate of human capital, which in turn depends on the time individuals use for acquiring skills.

However, the idea that human capital plays a major role in economic growth was not accepted by all. Several years ago there seemed to appear some kind of consensus among growth theorists, based on solid empirical evidence, that human capital plays a modest role in determining long-run rates of growth. Several recent papers have undermined this consensus. The results of Manuelli and Seshadri (2014) "suggest that human capital has a central role in determining the wealth of nations and that the quality of human capital varies systematically with the level of development". Illustrative accounting estimates by Jones (2014) "suggest that human capital variation can be substantially amplified, including to the point where capital variation could possibly fully account for cross-country income differences". Lucas (2015) argues that it rests on a misinterpretation of the evidence. He proposes "a very simple model of an economy that conforms well to the census evidence but in which all growth is driven by schooling and on-the-job learning".

Most of endogenous growth models with human capital accumulation assume a representative agent, which is only a fair approximation if income and wealth inequality play a negligible role in the process of economic development. However, it is widely recognized that inequality has a strong

\footnotetext{
${ }^{1}$ Adam Smith included in the capital stock of a nation the inhabitants' acquired and useful talents, and noticed that human skills increase wealth for society.
} 
impact on economic growth. The literature on the relationship between inequality and growth suggests different channels through which inequality can impact growth. On the one hand, the accumulation of savings (Stiglitz, 1969; Bourguignon, 1981), unobservable effort (Rebelo, 1991) and the investment project size (Barro, 2000) represent the main channels through which inequality may enhance growth. On the other hand, in the presence of credit market imperfections, inequality has a negative impact on investment in human capital accumulation (Galor and Zeira, 1993) or entrepreneurial activity (Banerjee and Newman, 1993), and hence on economic growth. Overall, the impact of inequality on growth depends on which channels dominate. Empirical studies also are generally inconclusive. While analyses by Alesina and Rodrik (1994), Persson and Tabellini (1994) and Perotti (1996) show a negative relationship between inequality and growth, more recent works by Partridge (1997), Forbes (2000) and Frank (2009) find a positive relationship. Barro (2000) finds that the effect of income inequality on economic growth may differ between poor and rich economies.

Economists have also paid some attention to the relationship between the accumulation of human capital and inequality. Becker and Tomes (1979), Viaene and Zilcha (2003) and Galor and Moav (2004) emphasize educational attainment as one of the causes of greater income inequality. Galor and Zeira (1993) and Banerjee and Newman (1993) identify credit market constraints as the channel relating the accumulation of human capital and inequality. Political considerations and education are considered in Saint-Paul and Verdier (1991), Glomm and Ravikumar (1992) and Eckstein and Zilcha (1994). Eicher and García-Peñalosa (2001) build a model, which predicts a non-monotonic relationship between educational attainment and inequality and explain the lack of a clear relation between inequality and growth. Most of these papers consider overlapping generations models in which the transfer of human capital across generations is an important factor of growth.

Turnovsky (2011) and Turnovsky and Mitra (2013) propose two-sector endogenous growth models ${ }^{2}$ linking human capital accumulation and income inequality. In those models agents are infinitely-lived and the heterogeneity of agents, which is the underlying source of income inequality, stems from their initial distribution of endowments of human capital. Their results suggest that an increase in the growth rate resulting from productivity enhancement in the human capital sector will be accompanied by an increase in inequality

\footnotetext{
${ }^{2}$ The sectors considered produce respectively the final output and human capital.
} 
whereas a productivity boost in the final output sector results in a reduction in inequality.

In practically all growth models with infinitely-lived agents, patience plays a key role. In exogenous growth models with physical capital, higher patience implies a higher propensity to save and hence a higher steady state stock of physical capital. In the context of endogenous growth models with human capital accumulation, higher patience implies greater incentives to devote time to the acquisition of skills and hence leads to higher rates of growth. Recent results of Hübner and Vannoorenberghe (2015) suggest that increasing patience by one standard deviation raises per-capita income by between $34 \%$ and $78 \%$. Dohmen et al. (2015) show that average patience explains a considerable fraction of the variation in growth rates both in the medium run and in the long run and about $40 \%$ of the between-country variation in income. Their results establish that, within countries, average patience in geographical regions predicts average years of education and, in individual-level analyses, that individual patience predicts educational attainment within countries and regions. Patience varies not only between countries, but also within countries. According to Falk et al. (2015), between-country variance accounts for about 13.5 percent of total variation in patience. Respectively, within-country variation accounts for about 86.5 percent of total variation.

There is a literature on models with infinitely-lived agents heterogeneous in their discount factor (see a very good survey by Becker (2006)). At the same time, to the best of our knowledge, all models with human capital accumulation assume either a representative agent or agents with an identical discount factor. In fact, the only type of heterogeneity considered in these models was heterogeneity in initial human capital. ${ }^{3}$ In this paper, we propose two endogenous growth models with human capital accumulation with heterogenous agents that differ in their discount factors, their skills in accumulating capital and initial human capital endowments. More precisely, in order to better understand the trade-off between inequalities and growth, we extend the Lucas' 1988 model introducing two classes of agents with heterogenous skills, patience and initial human wealth. ${ }^{4}$ We consider two regimes according to the planner's political constraints. In the first regime, that we call meritocracy, the planner faces individual constraints. In

\footnotetext{
${ }^{3}$ See Turnovsky (2011) and Turnovsky and Mitra (2013) cited above.

${ }^{4}$ Note that differently from the Ramsey model with physical capital accumulation (Becker, 1980), our extensions exhibit long-run distributions of capital (heterogenous human capital) that are non-degenerated.
} 
the second regime the planner faces an aggregate constraint, redistributing. We find that heterogeneity matters, specially with redistribution. Indeed, although in the meritocracy regime the solution coincides with the representative agent's Lucas BGP, in the redistribution case the optimal solution for non-leisure time devoted to capital accumulation is never interior for both agents. Either the less talented agents do not accumulate human capital or the more skilled agents do not work. A second important result is that social welfare under the redistribution regime is always higher than under meritocracy. Third, the redistribution of consumption is not affected by the distribution of skills, depending only on differences in patience. In contrast, the allocation of tasks takes into account skills differences. Finally we find that inequality in the distribution of human capital increases in time, and that inequality is associated with higher rates of growth in both regimes.

The rest of the paper is organized as follows. In the next section we present the fundamentals of the models considered. The "meritocracy" case and the "redistribution" cases are analyzed in Sections 3 and 4 respectively. In Section 5 we compare both cases and further discuss our results. Finally, in Section 6, we provide some concluding remarks. Proofs and computations are relegated to the Appendix.

\section{Fundamentals}

The models considered follow closely the Lucas (1988) framework, extending it to account for agents heterogeneity. Since we want to focus on the role of heterogeneity in human capital accumulation on inequality and growth, we ignore technological change and physical capital accumulation.

We consider two classes of agents: patient and impatient households, labeled respectively by 0 and 1 . They have different discount rates, $\rho_{i}, i=0,1$ with $\rho_{0}<\rho_{1}$.

We denote the individual labor supply by $l_{i t}$ and the size of each class by $\pi_{i}$. We normalize the size of the entire population to one so that $\pi_{0}+\pi_{1}=1$. Then, $l_{t}$, aggregate labor supply is given by

$$
l_{t}=\sum_{i=0}^{1} \pi_{i} l_{i t}
$$

We consider a linear constant returns technology. 
Assumption 1 Technology is represented by a production function

$$
y_{t}=A l_{t}
$$

where $y_{t}$ denotes aggregate production and $A>0$ is a scaling parameter.

Leisure time is exogenous. Non-leisure time is normalized to one and spent either working or accumulating human capital (education and health). Individual labor supply is given by the product of human capital, $h_{i t}$, and working time, $u_{i t}$, i.e.,

$$
l_{i t} \equiv h_{i t} u_{i t}
$$

This means that both factors are necessary in order to supply labor. The remaining non-leisure time, $1-u_{i t}$, is devoted to human capital accumulation.

The specification chosen for human capital accumulation of each class of agents is identical to the one considered in Lucas (1988). However we assume that the two classes have different skills in accumulating human capital.

Assumption 2 The law of human capital accumulation is given by

$$
\dot{h}_{i t} / h_{i t}=B_{i}\left(1-u_{i t}\right)
$$

If an individual does not devote any non-leisure time to human capital accumulation then there is no accumulation. If an individual devotes all his non-leisure time to human capital accumulation then his human capital grows at its maximal rate, $B_{i}$, which is specific to each class, denoting different skills. As in Lucas (1988) we assume that $B_{i}>\rho_{i}$.

We denote by $c_{i t}$ real consumption of an individual of class $i$, and by $c_{t}$ aggregate consumption in the economy. Production is entirely consumed so that $c_{t} \equiv \sum_{i=0}^{1} \pi_{i} c_{i t}=y_{t}$.

For simplicity we assume no labor or capital accumulation disutility so that:

Assumption 3 Preferences are rationalized by a logarithmic felicity

$$
\int_{0}^{\infty} e^{-\rho_{i} t} \ln c_{i t} d t
$$

with $\rho_{0}<\rho_{1}$. 
As in Lucas (1988) we consider a planner who maximizes the weighted sum of utilities

$$
\sum_{i=0}^{1} \pi_{i} \int_{0}^{\infty} e^{-\rho_{i} t} \ln c_{i t} d t=\int_{0}^{\infty} \sum_{i=0}^{1} \pi_{i} e^{-\rho_{i} t} \ln c_{i t} d t
$$

an intertemporal welfare functional, under (4). ${ }^{5}$

In the following, we consider two regimes according to the planner's political constraints. In the first regime, that we denote by "meritocracy", the planner's faces individual constraints:

$$
c_{i t} \leq A l_{i t}
$$

Remark that when instead of two classes of individuals the subscript $i$ denotes two countries, the meritocracy regime corresponds to the autarky situation.

In the second regime, that we call "redistribution", the planner faces an aggregate constraint:

$$
\sum_{i=0}^{1} \pi_{i} c_{i t} \leq A l_{t}
$$

When $i$ denotes countries instead of classes of individuals, this regime corresponds to the case where the two countries are integrated in one union.

\section{Meritocracy}

In this case the planner maximizes (6) subject to (4) and (7). The Hamiltonian writes:

$$
\sum_{i=0}^{1} \pi_{i} e^{-\rho_{i} t} \ln \left(A h_{i t} u_{i t}\right)+\sum_{i=0}^{1} \lambda_{i t} h_{i t} B_{i}\left(1-u_{i t}\right)
$$

We have two state variables $\left(h_{i t}\right)$ and two controls $\left(u_{i t}\right)$. The strict concavity of the objective function and the concavity of the law of motion with

\footnotetext{
${ }^{5}$ The question of time-consistency can be raised in the case of a welfare function maximization with heterogenous agents. As noted by Zuber (2011) and Heal and Millner (2015), stationarity and time-consistency of aggregate preferences hold together only if agents have the same discount factor.
} 
respect to $\left(h_{i t}, u_{i t}\right)$ satisfy the Arrow-Mangasarian sufficient condition for Hamiltonian maximization and imply the uniqueness of the planner's solution.

Proposition 1. The optimal solution is the Balanced Growth Path (BGP):

$$
u_{i t}^{*}=\frac{\rho_{i}}{B_{i}} \text { and } h_{i t}^{*}=h_{i 0} e^{\left(B_{i}-\rho_{i}\right) t}
$$

for $t \geq 0$. This solution is unique.

Proof. See the Appendix.

This solution coincides with that forwarded by Lucas (1988) in the representative agent's case. In fact, what the planner is doing in the meritocracy case is simply solving two independent problems, each of them featuring identical agents. Note that with the functional forms chosen we are able to prove analytically Lucas (1988) conjecture that the BGP is the optimal solution.

We can see that the equilibrium growth rate of human capital of class $i$ increases with the class ability in investing in human capital, $B_{i}$, and declines with increases in the class discount rate, $\rho_{i}$. However, what really matters for human capital growth is the difference between these two parameters. This means that more skilled but very impatient agents may accumulate human capital at a lower rate than less skilled but more patient ones. Since this outcome is not supported by empirical studies, we rule out this possibility, assuming that the growth rate of human capital accumulation of the patient consumer, $B_{0}-\rho_{0}$, always exceeds the growth case of the impatient one, $B_{1}-\rho_{1}$, i.e. we consider that the patient consumer, $i=0$, is the dominant one. Let $\delta \equiv\left(B_{0}-\rho_{0}\right)-\left(B_{1}-\rho_{1}\right)$ denote the difference between the growth rates of patient and impatient consumers. We assume that:

Assumption $4 \delta>0$.

\subsection{Inequality and growth in the meritocracy case}

Let us now discuss the trade-off between social inequalities and growth in the meritocracy case. The first choice that we have to make is how to measure these two concepts. Most studies use income inequality data as a proxy for social inequality and the growth rate of income to measure economic growth. In this work we chose to measure social inequalities using the Gini 
index of consumption, while economic growth is measured by the growth rate of aggregate consumption. In the meritocracy case as consumption and income (output) coincide, both at the individual and aggregate level, this choice is irrelevant. In contrast, in the redistribution case, although aggregate consumption is still identical to aggregate output, the real welfare of each individual is given by consumption and not by output. Therefore, social inequalities are better proxied using a measure of consumption inequality. Also, since in this work we focus on the effects of human capital on inequality and growth we start by presenting the Gini index of human wealth, which measures inequality in the distribution of human capital.

Proposition 2. If, without loss of generality, $h_{0 t}>h_{1 t}$, the period $t$ Gini index of human wealth is given by

$$
g_{h t}=\pi_{1}-\frac{\pi_{1} h_{1 t}}{\pi_{0} h_{0 t}+\pi_{1} h_{1 t}}
$$

Proof. See the Appendix.

The stock of human capital that a class has accumulated until period $t$, $h_{i t}$, depends not only on the class rate of growth of human capital accumulation, $B_{i}-\rho_{i}$, but also on its initial human capital endowment, $h_{i 0}$. However, it is easy to see that, in the long run, the growth rate effect will dominate. We have therefore the following proposition.

Proposition 3 (human wealth inequality). Let Assumption 4 hold.

1. If $h_{00}>h_{10}$, then $h_{0 t}>h_{1 t}$ for every $t$ and

$$
g_{h t}=\pi_{1}-\frac{\pi_{1} h_{10} e^{-\delta t}}{\pi_{0} h_{00}+\pi_{1} h_{10} e^{-\delta t}}
$$

Thus, $g_{t}$ increases monotonically from $g_{h 0}=\pi_{1}-\pi_{1} h_{10} /\left(\pi_{0} h_{00}+\pi_{1} h_{10}\right)$ to $g_{\infty}=\pi_{1}$.

2. If $h_{00}<h_{10}$, then there is a critical date

$$
T_{h}=\frac{\ln \left(h_{10} / h_{00}\right)}{\delta}
$$


beyond which the human capital stock of the dominant (patient) class exceeds the human capital stock of the impatient class. In this case, the Gini index of human wealth is given by

$$
\begin{aligned}
g_{h t} & =\pi_{0}-\frac{\pi_{0} h_{00}}{\pi_{0} h_{00}+\pi_{1} h_{10} e^{-\delta t}} \text { for } t \leq T_{h} \\
g_{h t} & =\pi_{1}-\frac{\pi_{1} h_{10} e^{-\delta t}}{\pi_{0} h_{00}+\pi_{1} h_{10} e^{-\delta t}} \text { for } t>T_{h}
\end{aligned}
$$

Proof. See the Appendix.

Since human capital and consumption of an individual of type $i$ grow at the same rate along the BGP, the observed differences in the evolution of the Gini index of human wealth and the Gini index of consumption reflect mainly differences in initial endowments, being therefore relevant only in the short run.

Definition 4. If, without loss of generality, $c_{0 t}>c_{1 t}$, the Gini index of consumption is given by

$$
g_{c t}=\pi_{1}-\frac{\pi_{1} c_{1 t}}{\pi_{0} c_{0 t}+\pi_{1} c_{1 t}}
$$

Proposition 5 (consumption inequality). Let Assumption 4 hold.

1. If $c_{00}>c_{10}$, then $c_{0 t}>c_{1 t}$ for every $t$ and

$$
g_{c t}=\pi_{1}-\frac{\pi_{1} c_{10} e^{-\delta t}}{\pi_{0} c_{00}+\pi_{1} c_{10} e^{-\delta t}}
$$

Thus, $g_{t}$ increases monotonically from $g_{0}=\pi_{1}-\pi_{1} c_{10} /\left(\pi_{0} c_{00}+\pi_{1} c_{10}\right)$ to $g_{\infty}=\pi_{1}$.

2. If $c_{00}<c_{10}$, then there is a critical date

$$
T_{c}=\frac{\ln \left(c_{10} / c_{00}\right)}{\delta}
$$

beyond which consumption of the dominant (patient) class exceeds consumption of the impatient class. In this case, the Gini index of consumption is given by

$$
\begin{aligned}
& g_{c t}=\pi_{0}-\frac{\pi_{0} c_{00}}{\pi_{0} c_{00}+\pi_{1} c_{10} e^{-\delta t}} \text { for } t \leq T_{c} \\
& g_{c t}=\pi_{1}-\frac{\pi_{1} c_{10} e^{-\delta t}}{\pi_{0} c_{00}+\pi_{1} c_{10} e^{-\delta t}} \text { for } t>T_{c}
\end{aligned}
$$


Proof. See the Appendix.

Remark that $h_{00}>h_{10}$ does not imply $c_{00}>c_{10}$. Indeed, using (3), (7) and (9), we can rewrite this last inequality as

$$
h_{00}>h_{10} \frac{\rho_{1}}{\rho_{0}} \frac{B_{0}}{B_{1}}
$$

where $\rho_{1} / \rho_{0}>1$, so that we have $c_{10}>c_{00}$ if $B_{1}$ is sufficiently low.

We now describe the evolution of the growth rate of aggregate consumption.

Proposition 6 (consumption growth rate). The dynamics of the aggregate consumption growth rate are given by

$$
\gamma_{c t} \equiv \frac{\dot{c}_{t}}{c_{t}}=\frac{\pi_{0} c_{00}\left(B_{0}-\rho_{0}\right) e^{\delta t}+\pi_{1} c_{10}\left(B_{1}-\rho_{1}\right)}{\pi_{0} c_{00} e^{\delta t}+\pi_{1} c_{10}}
$$

Proof. See the Appendix.

We can now characterize the trade-off between inequality and growth in the meritocracy case.

Proposition 7 (trade-off inequality-growth). Let Assumption 4 hold.

In case (1) of Proposition $5\left(c_{00}>c_{10}\right)$, the higher the consumption inequality, the higher the growth rate:

$$
\gamma_{c}=\pi_{0}\left(B_{0}-\rho_{0}\right)+\pi_{1}\left(B_{1}-\rho_{1}\right)+\delta g_{c}
$$

In case (2) of Proposition $5\left(c_{00}<c_{10}\right)$, this trade-off holds after $T_{c}$ and is reversed before:

$$
\begin{aligned}
& \gamma_{c}=\pi_{0}\left(B_{0}-\rho_{0}\right)+\pi_{1}\left(B_{1}-\rho_{1}\right)-\delta g_{c} \text { for } t \leq T_{c} \\
& \gamma_{c}=\pi_{0}\left(B_{0}-\rho_{0}\right)+\pi_{1}\left(B_{1}-\rho_{1}\right)+\delta g_{c} \text { for } t>T_{c}
\end{aligned}
$$

Proof. See the Appendix.

We conclude that, in the long run, inequality always promotes growth in the meritocracy case. However, in the short run, depending on the initial distribution of skills and endowments, this result may be reversed. Indeed, in the subcase $t \leq T_{c}$ of case (2) where $c_{00}<c_{10}$, the lower the social inequality, the higher the growth rate. 


\section{Redistribution}

In this case the planner maximizes the same social welfare functional:

$$
\sum_{i=0}^{1} \pi_{i} \int_{0}^{\infty} e^{-\rho_{i} t} \ln c_{i t} d t=\int_{0}^{\infty} \sum_{i=0}^{1} \pi_{i} e^{-\rho_{i} t} \ln c_{i t} d t
$$

under the resource constraint

$$
\sum_{i=0}^{1} \pi_{i} c_{i t} \leq A \sum_{i=0}^{1} \pi_{i} h_{i t} u_{i t}
$$

and the law of motion of human capital

$$
\dot{h}_{i t}=B_{i}\left(1-u_{i t}\right) h_{i t}
$$

with $0 \leq u_{i t} \leq 1$.

There are two state variables $\left(h_{i t}\right)$ and four controls $\left(c_{i t}\right.$ and $\left.u_{i t}\right)$. In this case, since there is redistribution, the planner can solve its problem in two stages. In the first stage, given $h_{i t}$ and $u_{i t}$, the planner solves a static problem:

$$
\max _{c_{0 t}, c_{1 t}} \sum_{i=0}^{1} \pi_{i} e^{-\rho_{i} t} \ln c_{i t}
$$

subject to (24). The solution of this static program is given by:

$$
c_{i t}=A e^{-\rho_{i} t} \frac{\sum_{j=0}^{1} \pi_{j} h_{j t} u_{j t}}{\sum_{j=0}^{1} \pi_{j} e^{-\rho_{j} t}}
$$

Note that the division of consumption among individuals is only determined by the degree of impatient, $\rho_{i}$, not being influenced by the distribution of skills, $B_{i}$. Since $c_{t}=y_{t}$, using (24) and (25) we obtain the consumption share

$$
s_{i t} \equiv \frac{\pi_{i} c_{i t}}{c_{t}}=\frac{\pi_{i} e^{-\rho_{i} t}}{\sum_{j=0}^{1} \pi_{j} e^{-\rho_{j} t}}
$$

Substituting now (25) in the original problem it becomes

$$
\max \int_{0}^{\infty}\left[\sum_{i=0}^{1} \pi_{i} e^{-\rho_{i} t} \ln \frac{A e^{-\rho_{i} t}}{\sum_{j=0}^{1} \pi_{j} e^{-\rho_{j} t}}+\left(\sum_{i=0}^{1} \pi_{i} e^{-\rho_{i} t}\right) \ln \sum_{j=0}^{1} \pi_{j} h_{j t} u_{j t}\right] d t
$$


or, equivalently,

$$
\max \int_{0}^{\infty}\left(\sum_{i=0}^{1} \pi_{i} e^{-\rho_{i} t}\right) \ln \sum_{j=0}^{1} \pi_{j} h_{j t} u_{j t} d t
$$

subject to $\dot{h}_{i t}=B_{i}\left(1-u_{i t}\right) h_{i t}$ with $0 \leq u_{i t} \leq 1$.

We now have to obtain the optimal solution for $h_{i t}$ and $u_{i t}$. To better understand the mechanisms involved we will start by analyzing the case where $B_{0}=B_{1}$, the only difference between the two types of agents being their degree of patience.

\subsection{Redistribution without heterogeneity in skills}

In this case $B_{0}=B_{1}=B$, and $0<\rho_{0}<\rho_{1}$.

Proposition 8. If $B_{0}=B_{1}=B$, and $0<\rho_{0}<\rho_{1}$ the optimal trajectory $\left(h_{i t}, u_{i t}\right)_{i=0,1}$ is given by:

$$
h_{i t}=\phi \zeta_{i} \sum_{j=0}^{1} \frac{\pi_{j}}{\rho_{j}} e^{\left(B-\rho_{j}\right) t} \text { and } u_{i t}=\frac{1}{B} \frac{\sum_{j=0}^{1} \pi_{j} e^{\left(B-\rho_{j}\right) t}}{\sum_{j=0}^{1} \frac{\pi_{j}}{\rho_{j}} e^{\left(B-\rho_{j}\right) t}} \in(0,1)
$$

for $t \geq 0$, where

$$
\phi=\frac{\sum_{i=0}^{1} \pi_{i} h_{i 0}}{\sum_{i=0}^{1} \frac{\pi_{i}}{\rho_{i}}} \text { and } \zeta_{i}=\frac{h_{i 0}}{\sum_{j=0}^{1} \pi_{j} h_{j 0}}
$$

Proof. See the Appendix.

Observe that in this case $\left(B_{0}=B_{1}=B\right)$, we have $u_{0 t}=u_{1 t}=u_{t}$, which converges to $\rho_{0} / B$. The total human capital stock $H_{t}=\sum_{i=0}^{1} \pi_{i} h_{i t}$, satisfies

$$
\dot{H}_{t}=B H_{t}\left(1-u_{t}\right)
$$

\subsection{Redistribution with heterogeneity in skills}

In this subsection we obtain the optimal trajectory $\left(h_{i t}, u_{i t}\right)_{i=0,1}$ for $B_{0} \neq B_{1}$, and $0<\rho_{0}<\rho_{1}$.

Our first important result is given in the Lemma below. 
Lemma 9 (no interior solution). Let $B_{0} \neq B_{1}$. For every $t$, there exists an $i$ such that $u_{i t} \in\{0,1\}$.

Lemma 9 tells us that, in contrast to the meritocracy case and to the redistribution case without heterogeneity in skills, we never have an interior solution for working time, $u_{i t}$, of both classes of agents.

Using this lemma, we can find the explicit planner's solution, which is the optimal trajectory. To this purpose we introduce the following assumption.

Assumption $5 B_{0}>B_{1}>\rho_{1}>\rho_{0}$.

According to Assumption 5 the dominant class is not only more patient $\left(\rho_{0}<\rho_{1}\right)$, but is also more talented in accumulating human capital $\left(B_{0}>\right.$ $\left.B_{1}\right)$. Assumption 5 is of course more restrictive than Assumption 4. Indeed, Assumption 5 implies $\delta>0$, while the reverse is not true.

Let us define the average discount rate, $\rho(t)$, as a time-dependent harmonic mean of discount rates

$$
\rho(t) \equiv\left(\sum_{i=0}^{1} \frac{1}{\rho_{i}} \frac{\pi_{i} e^{-\rho_{i} t}}{\sum_{j=0}^{1} \pi_{j} e^{-\rho_{j} t}}\right)^{-1} \in\left(\rho_{0}, \rho_{1}\right)
$$

Of course, under Assumption 5, $B_{0}>B_{1}>\rho(t)$. Moreover, $\rho(t)$ decreases from $\rho(0) \in\left(\rho_{0}, \rho_{1}\right)$ to $\rho(\infty)=\rho_{0}$. If $t=0,(28)$ simplifies to:

$$
\rho(0)=\left(\sum_{i=0}^{1} \frac{\pi_{i}}{\rho_{i}}\right)^{-1}
$$

which is a harmonic mean where the weights are the size of each class, $\pi_{i}$.

In the following,

$$
H_{i t} \equiv \pi_{i} h_{i t} \text { and } \sigma_{i t} \equiv \frac{H_{i t}}{H_{0 t}+H_{1 t}}
$$

will denote respectively the human capital of class $i$ at time $t$ and its aggregate share. Of course, $\sum_{i=0}^{1} \sigma_{i t}=1$.

To simplify the presentation we will consider separately the following two cases:

1. $\sigma_{10} \leq \sigma_{10}^{*} \equiv \rho(0) / B_{0}$,

2. $\sigma_{10}>\sigma_{10}^{*}$.

Note that in the first case, since $\sigma_{10}$ is sufficiently small, the more patient and talented class is also relatively well endowed in initial human capital. 


\subsubsection{The case where $\sigma_{10} \leq \sigma_{10}^{*}$}

The optimal trajectory in this case is given in Proposition 10 below.

Proposition 10. Let Assumption 5 hold and assume that $\sigma_{10} \leq \sigma_{10}^{*} \equiv$ $\rho(0) / B_{0}$. Then, the optimal trajectory $\left(h_{i t}, u_{i t}\right)_{i=0,1}$ is given by

$$
\begin{aligned}
& h_{0 t}=\frac{H_{10}}{\sigma_{10}} \frac{\rho(0)}{\pi_{0}} \sum_{i=0}^{1} \pi_{i}\left[\frac{e^{\left(B_{0}-\rho_{i}\right) t}}{\rho_{i}}-\frac{\sigma_{10}}{\rho_{i}}\right] \\
& u_{0 t}=\frac{\sum_{i=0}^{1} \pi_{i}\left[\frac{e^{\left(B_{0}-\rho_{i}\right) t}}{B_{0}}-\frac{\sigma_{10}}{\rho_{i}}\right]}{\sum_{i=0}^{1} \pi_{i}\left[\frac{e^{\left(B_{0}-\rho_{i}\right) t}}{\rho_{i}}-\frac{\sigma_{10}}{\rho_{i}}\right]} \in(0,1)
\end{aligned}
$$

and

$$
\begin{aligned}
& h_{1 t}=h_{10} \\
& u_{1 t}=1
\end{aligned}
$$

for every $t \geq 0$.

Proof. See the Appendix.

We notice that $0<u_{0 t}<1$ and $u_{1 t}=1$ for every $t \geq 0$. This means that when the initial human capital endowment of the patient and talented class is relatively important, the less patient and less talented class never invests in human capital, devoting all its non-leisure time to work. This result can be seen as an application of the comparative advantage principle, according to which agents will specialize in the activity where they are relatively better. However, the more patient and talented agent also devotes some non-leisure to work every period. Remember that in order to supply labor, contributing therefore to production, an agent must devote some time to work. See (3). In this case, as the initial capital endowment of the skilled class is sufficiently important, these agents divide each period their non-leisure time between work and capital accumulation, contributing to the production effort since the beginning. Also, since, under Assumption 5, $B_{0}>\rho_{1}$, we get $\lim _{t \rightarrow \infty} u_{0 t}=$ $\rho_{0} / B_{0}<1$.

It is easy to conclude that since only the more talented and patient class accumulates human capital, its human capital share, $\sigma_{0 t}$, strictly increases in time. Replacing (30) and (32) in (29) we obtain the dynamics of the human capital shares. 
Lemma 11 (shares of human capital). Under Assumption 5 and $\sigma_{10} \leq \sigma_{10}^{*} \equiv$ $\rho(0) / B_{0}$, the shares of human capital at time $t$ are given by

$$
\begin{aligned}
\sigma_{0 t} & =1-\sigma_{1 t} \\
\sigma_{1 t} & =\frac{\sigma_{10}}{\sum_{i=0}^{1} w_{i} e^{\left(B_{0}-\rho_{i}\right) t}}
\end{aligned}
$$

for any $t$, where the denominator is an average with weights

$$
w_{i} \equiv \frac{\pi_{i}}{\rho_{i}} / \sum_{j=0}^{1} \frac{\pi_{j}}{\rho_{j}}
$$

\subsubsection{The case where $\sigma_{10}>\sigma_{10}^{*}$}

We start by introducing four important critical values: $T_{0}, T_{1}, T_{2}$, with $T_{1}<T_{2}$, and $\lambda_{10}$. We define $T_{0}$ and $T_{2}\left(\lambda_{10}\right)$ respectively as the solutions of the two following equalities below

$$
\begin{aligned}
& \sigma_{1 T_{0}}=\sigma_{1 T_{0}}^{*} \equiv \frac{\rho\left(T_{0}\right)}{B_{0}} \\
& \sigma_{1 T_{2}}=\sigma_{1 T_{2}}^{*} \equiv \frac{\rho\left(T_{2}\right)}{B_{0}}
\end{aligned}
$$

that we can rewrite respectively as:

$$
\begin{aligned}
\frac{B_{0}}{\rho\left(T_{0}\right)} & =1+\frac{H_{00}}{H_{10}} e^{B_{0} T_{0}} \\
\frac{B_{0}}{\rho\left(T_{2}\right)} & =1+\frac{H_{00}}{H_{10}} \frac{e^{B_{0} T_{2}}}{e^{B_{1} T_{1}}}\left[1+\frac{\sum_{i=0}^{1} \frac{\pi_{i}}{\rho_{i}}\left(1-e^{-\rho_{i} T_{1}}\right)}{\sum_{i=0}^{1} \frac{\pi_{i}}{B_{1}} e^{-\rho_{i} T_{1}}}\right]
\end{aligned}
$$

where $T_{1}\left(\lambda_{10}\right)$ is the smallest solution of

$$
\lambda_{10} h_{10}=\sum_{i=0}^{1} \frac{\pi_{i}}{B_{1}} e^{-\rho_{i} T_{1}}+\sum_{i=0}^{1} \frac{\pi_{i}}{\rho_{i}}\left(1-e^{-\rho_{i} T_{1}}\right)
$$

Note that at $T_{1}$ we have $u_{1 t}=1$. Notice also that $T_{2}$ equals $T_{0}$ when $T_{1}=0$. Finally, let $\lambda_{10}$ be the solution of

$$
f\left(\lambda_{10}\right) \equiv \frac{\frac{1}{\rho\left(T_{2}\left(\lambda_{10}\right)\right)}-\frac{1}{B_{0}}}{\frac{1}{\rho\left(T_{1}\left(\lambda_{10}\right)\right)}-\frac{1}{B_{1}}}-\frac{\sum_{i=0}^{1} \pi_{i} e^{-\rho_{i} T_{1}\left(\lambda_{10}\right)}}{\sum_{i=0}^{1} \pi_{i} e^{-\rho_{i} T_{2}\left(\lambda_{10}\right)}}=0
$$


$\lambda_{10}$ determines in turn $T_{1}\left(\lambda_{10}\right)$ and $T_{2}\left(\lambda_{10}\right)$.

The optimal trajectory in this case, where $\sigma_{10}>\sigma_{10}^{*}$, i.e., the initial human capital share of the less patient and less talented class exceeds the critical threshold $\sigma_{10}^{*} \equiv \rho(0) / B_{0}$, is given in Proposition 12 below.

Proposition 12. Let Assumption 5 hold and assume that $\sigma_{10}>\sigma_{10}^{*} \equiv$ $\rho(0) / B_{0}$.

1. If

$$
B_{1} \leq B_{1}^{*} \equiv \frac{1}{\frac{1}{\rho(0)}-\left[\frac{1}{\rho\left(T_{0}\right)}-\frac{1}{B_{0}}\right] \sum_{i=0}^{1} \pi_{i} e^{-\rho_{i} T_{0}}}
$$

then the optimal trajectory $\left(h_{i t}, u_{i t}\right)_{i=0,1}$ is given by

$$
\begin{aligned}
h_{0 t} & =h_{00} e^{B_{0} t} \text { for } 0 \leq t \leq T_{0} \\
h_{0 t} & =\frac{H_{10}}{\pi_{0}}\left[\frac{\sum_{i=0}^{1} \frac{\pi_{i}}{\rho_{i}} e^{\left(B_{0}-\rho_{i}\right) t}}{\sum_{i=0}^{1} \frac{\pi_{i}}{B_{0}} e^{\left(B_{0}-\rho_{i}\right) T_{0}}}-1\right] \text { for } t>T_{0} \\
u_{0 t} & =0 \text { for } 0 \leq t \leq T_{0} \\
u_{0 t} & =\frac{\sum_{i=0}^{1} \frac{\pi_{i}}{B_{0}} e^{\left(B_{0}-\rho_{i}\right) t}-\sum_{i=0}^{1} \frac{\pi_{i}}{B_{0}} e^{\left(B_{0}-\rho_{i}\right) T_{0}}}{\sum_{i=0}^{1} \frac{\pi_{i}}{\rho_{i}} e^{\left(B_{0}-\rho_{i}\right) t}-\sum_{i=0}^{1} \frac{\pi_{i}}{B_{0}} e^{\left(B_{0}-\rho_{i}\right) T_{0}}} \in(0,1) \text { for } t>T(4)
\end{aligned}
$$

and

$$
\begin{aligned}
& h_{1 t}=h_{10} \text { for any } t \\
& u_{1 t}=1 \text { for any } t
\end{aligned}
$$

2. If

$$
B_{1}>B_{1}^{*} \equiv \frac{1}{\frac{1}{\rho(0)}-\left[\frac{1}{\rho\left(T_{0}\right)}-\frac{1}{B_{0}}\right] \sum_{i=0}^{1} \pi_{i} e^{-\rho_{i} T_{0}}}
$$

then the optimal trajectory $\left(h_{i t}, u_{i t}\right)_{i=0,1}$ is given by

$$
\begin{aligned}
& h_{0 t}=h_{00} e^{B_{0} t} \text { for } 0 \leq t \leq T_{2} \\
& h_{0 t}=\frac{H_{1 T_{2}}}{\pi_{0}}\left[\frac{\sum_{i=0}^{1} \frac{\pi_{i}}{\rho_{i}} e^{\left(B_{0}-\rho_{i}\right) t}}{\lambda_{1 T_{2}} h_{1 T_{2}} e^{B_{0} T_{2}}}-1\right] \text { for } t \geq T_{2} \\
& u_{0 t}=0 \text { for } 0 \leq t \leq T_{2} \\
& u_{0 t}=\frac{\sum_{i=0}^{1} \frac{\pi_{i}}{B_{0}} e^{\left(B_{0}-\rho_{i}\right) t}-\lambda_{1 T_{2}} h_{1 T_{2}} e^{B_{0} T_{2}}}{\sum_{i=0}^{1} \frac{\pi_{i}}{\rho_{i}} e^{\left(B_{0}-\rho_{i}\right) t}-\lambda_{1 T_{2}} h_{1 T_{2}} e^{B_{0} T_{2}}} \in(0,1) \text { for } t \geq T_{2}
\end{aligned}
$$


and

$$
\begin{aligned}
h_{1 t} & =h_{10} e^{B_{1} t}\left[1-\frac{1}{\lambda_{10} h_{10}} \sum_{i=0}^{1} \frac{\pi_{i}}{\rho_{i}}\left(1-e^{-\rho_{i} t}\right)\right] \text { for } 0 \leq t \leq T_{1}(49) \\
h_{1 t} & =h_{1 T_{1}} \text { for } t \geq T_{1} \\
u_{1 t} & =\frac{1}{B_{1}} \frac{\sum_{i=0}^{1} \pi_{i} e^{-\rho_{i} t}}{\lambda_{10} h_{10}+\sum_{i=0}^{1} \frac{\pi_{i}}{\rho_{i}}\left(e^{-\rho_{i} t}-1\right)} \in(0,1) \text { for } 0 \leq t<T_{1}(50) \\
u_{1 t} & =1 \text { for } t \geq T_{1}
\end{aligned}
$$

where $\lambda_{10} h_{10}$ is given by (37),

$$
\lambda_{1 T_{2}} h_{1 T_{2}}=\sum_{i=0}^{1} \frac{\pi_{i}}{B_{1}} e^{-\rho_{i} T_{1}}+\sum_{i=0}^{1} \frac{\pi_{i}}{\rho_{i}}\left(e^{-\rho_{i} T_{2}}-e^{-\rho_{i} T_{1}}\right)
$$

and $H_{1 T_{2}}=\pi_{1} h_{1 T_{1}}$.

Proof. See the Appendix.

In case 1 of Proposition (12), although the initial capital share of the less talented and less patient individuals is now above the critical threshold $\rho(0) / B_{0}$, as they are not sufficiently skilled, $B_{1} \leq B_{1}^{*}$, these individuals still never accumulate human capital, devoting all their non-leisure time to work, i.e. we have, as in the previous case, $h_{1 t}=h_{10}$ and $u_{1 t}=1$ for any $t$. However, now the dominant class does not work initially, devoting all their non-leisure time to capital accumulation, that, until period $T_{0}$ increases at the maximal accumulation rate, $B_{0}$. See (42). Therefore, in this case for $t<T_{0}$, specialization is more intense: the less talented agents just work, while the others only accumulate capital. Nevertheless, once the share of human capital of the more talented class reaches the critical level $1-\rho(t) / B_{0}$, i.e. when $\sigma_{1 T_{0}}=\sigma_{1 T_{0}}^{*}$, the dominant class starts working and, since less time is devoted to capital accumulation, the rate of growth of human capital decreases over time.

As the less talented agents never accumulate capital $H_{1 t}=H_{10}$ for any $t$. Then, we get immediately the following result. 
Lemma 13 (shares of human capital). In case 1 of Proposition (12), the shares of human capital at time $t$ are given by

$$
\begin{aligned}
\sigma_{0 t} & =1=\sigma_{1 t} \text { for any } t \geq 0 \\
\sigma_{1 t} & =\frac{\sigma_{10}}{\sigma_{10}+\left(1-\sigma_{10}\right) e^{B_{0} t}} \text { for } 0 \leq t \leq T_{0} \\
\sigma_{1 t} & =\frac{\sigma_{1 T_{0}}}{\sum_{i=0}^{1} w_{i} e^{\left(B_{0}-\rho_{i}\right)\left(t-T_{0}\right)}} \text { for } t>T_{0}
\end{aligned}
$$

where the denominator in (52) is an average with weights

$$
w_{i} \equiv \frac{\frac{\pi_{i}}{\rho_{i}} e^{\left(B_{0}-\rho_{i}\right) T_{0}}}{\sum_{j=0}^{1} \frac{\pi_{j}}{\rho_{j}} e^{\left(B_{0}-\rho_{j}\right) T_{0}}}
$$

and

$$
\sigma_{1 T_{0}}=\frac{\sum_{i=0}^{1} \frac{\pi_{i}}{B_{0}} e^{\left(B_{0}-\rho_{i}\right) T_{0}}}{\sum_{i=0}^{1} \frac{\pi_{i}}{\rho_{i}} e^{\left(B_{0}-\rho_{i}\right) T_{0}}}
$$

We observe that (34) is a particular case of (53) with $T_{0}=0$.

In case 2 of Proposition (12), since the starting value of the capital share of the more talented class is below $1-\sigma_{10}^{*}$, again the dominant class does not work initially, only accumulating capital, which until period $T_{2}$, where $\sigma_{0 T_{2}}$ reaches the critical value $1-\rho\left(T_{2}\right) / B_{0}$, increases at the maximal accumulation rate, $B_{0}$. See (46). As in the previous case, after date $T_{2}$ the more talented agents start working and accumulate capital at a lower pace. However, as, in this case, the more impatient agents are more skilled in accumulating capital, they now accumulate capital until period $T_{1}$, dividing their non-leisure time between capital accumulation and work. Note however that the time they devote to capital accumulation decreases continuously from period 0 to $T_{1}$, where $u_{1 T_{1}}=1$. After period $T_{1}$ they stop accumulating capital, devoting all their non-leisure time to work. As $T_{1} \leq T_{2}$ and until $T_{2}$ the other class is accumulating capital at the maximal rate, their capital share declines steadily until period $T_{1}$. The evolution in time of the capital shares of both classes is given below in Lemma 14 . 
Lemma 14. In case 2 of Proposition (12), the shares of human capital at time $t$ are given by

$$
\begin{aligned}
\sigma_{0 t} & =1-\sigma_{1 t} \text { for any } t \geq 0 \\
\sigma_{1 t} & =\frac{\sigma_{10}}{\sigma_{10}+\left(1-\sigma_{10}\right) \frac{\lambda_{10} h_{10} e^{\left(B_{0}-B_{1}\right) t}}{\lambda_{10} h_{10}-\sum_{i=0}^{1} \frac{\pi_{i}}{\rho_{i}}\left(1-e^{-\rho_{i} t}\right)}} \text { for } 0 \leq t \leq T_{1} \\
\sigma_{1 t} & =\frac{\sigma_{1 T_{1}}}{\sigma_{1 T_{1}}+\left(1-\sigma_{1 T_{1}}\right) e^{B_{0}\left(t-T_{1}\right)}} \text { for } T_{1} \leq t \leq T_{2} \\
\sigma_{1 t} & =\frac{\sigma_{1 T_{2}}}{\sum_{i=0}^{1} w_{i} e^{\left(B_{0}-\rho_{i}\right)\left(t-T_{2}\right)}} \text { for } t \geq T_{2}
\end{aligned}
$$

where $\lambda_{10} h_{10}$ is given by (37) and the denominator in (54) is an average with weights

$$
w_{i} \equiv \frac{\frac{\pi_{i}}{\rho_{i}} e^{\left(B_{0}-\rho_{i}\right) T_{2}}}{\sum_{j=0}^{1} \frac{\pi_{j}}{\rho_{j}} e^{\left(B_{0}-\rho_{j}\right) T_{2}}}
$$

and

$$
\sigma_{1 T_{2}}=\frac{\lambda_{1 T_{2}} h_{1 T_{2}} e^{B_{0} T_{2}}}{\sum_{i=0}^{1} \frac{\pi_{i}}{\rho_{i}} e^{\left(B_{0}-\rho_{i}\right) T_{2}}}
$$

\subsubsection{Summarizing interpretation}

In this section we considered two classes with different skills, different degrees of patience and different initial capital stocks. We assumed, in line with empirical plausibility, that the more talented class is also more patient. We showed that in this case we never have an interior solution for working time of both classes of agents. Moreover, several optimal solutions exist, depending on the initial distribution of human capital and on the level of skills of the less talented class. If the initial human capital share of the less skilled individuals is not high enough, they will never accumulate human capital, devoting all their non-leisure time to work. If their initial human capital share is sufficiently high two cases are possible. Either they are not sufficiently skilled and again they only work, never accumulating human capital, or they are sufficiently skilled and devote initially some of their non-leisure time to human capital accumulation. However, the time they devote to human capital accumulation decreases steadily in time, so that in the long run we find that, as before, the less talented agents never accumulate human capital. In contrast, the more talented individuals always accumulate human capital. 
When their initial human capital share exceeds a critical value, these individuals will also work every period. However, if their initial share of human capital is not high enough, they will not work initially, devoting all their non-leisure time to capital accumulation, that grows at the maximal possible rate. This behavior continues until this critical level of their capital share is reached. After, they will start working and accumulating capital as in the previous solution. It is interesting to note two things. First, what matters for the choice of the optimal solution is the distribution of human capital and not its level. ${ }^{6}$ Second, in the long run, independently of the initial conditions and of the level of skills, we always get the same result: less talented individuals never accumulate capital and the more skilled agents work and accumulate capital.

\subsection{Inequality and growth in the redistribution case}

Let us now discuss the trade-off between social inequalities and growth in the redistribution case with heterogenous skills and discounting. From Lemmas 11,13 , and 14, we obtain immediately the dynamics of the Gini index of wealth in this case.

Proposition 15 (human wealth inequality). If, without loss of generality, $h_{0 t}>h_{1 t}$, the Gini index of human wealth is given by

$$
g_{h t}=\pi_{1}-\sigma_{1 t}
$$

The dynamics of wealth inequality are the following.

When $\sigma_{10} \leq \sigma_{10}^{*}$ :

$$
g_{h t}=\pi_{1}-\frac{\sigma_{10}}{\sum_{i=0}^{1} w_{i} e^{\left(B_{0}-\rho_{i}\right) t}} \text { for any } t \geq 0
$$

When $\sigma_{10}>\sigma_{10}^{*}$ and $B_{1} \leq B_{1}^{*}$ :

$$
\begin{aligned}
& g_{h t}=\pi_{1}-\frac{\sigma_{10}}{\sigma_{10}+\left(1-\sigma_{10}\right) e^{B_{0} t}} \text { for } 0 \leq t \leq T_{0} \\
& g_{h t}=\pi_{1}-\frac{\sigma_{1 T_{0}}}{\sum_{i=0}^{1} w_{i} e^{\left(B_{0}-\rho_{i}\right)\left(t-T_{0}\right)}} \text { for } t>T_{0}
\end{aligned}
$$

\footnotetext{
${ }^{6}$ Remark that the critical share of one class is always equal to one minus the critical share of the other class.
} 
When $\sigma_{10}>\sigma_{10}^{*}$ and $B_{1}>B_{1}^{*}$ :

$$
\begin{aligned}
& g_{h t}=\pi_{1}-\frac{\sigma_{10}}{\sigma_{10}+\left(1-\sigma_{10}\right) \frac{\lambda_{10} h_{10} e^{\left(B_{0}-B_{1}\right) t}}{\lambda_{10} h_{10}-\sum_{i=0}^{1} \frac{\pi_{i}}{\rho_{i}}\left(1-e^{-\rho_{i} t}\right)}} \text { for } 0 \leq t \leq T_{1} \\
& g_{h t}=\pi_{1}-\frac{\sigma_{1 T_{1}}}{\sigma_{1 T_{1}}+\left(1-\sigma_{1 T_{1}}\right) e^{B_{0}\left(t-T_{1}\right)}} \text { for } T_{1} \leq t \leq T_{2} \\
& g_{h t}=\pi_{1}-\frac{\sigma_{1 T_{2}}}{\sum_{i=0}^{1} w_{i} e^{\left(B_{0}-\rho_{i}\right)\left(t-T_{2}\right)}} \text { for } t \geq T_{2}
\end{aligned}
$$

In any case, the optimal Gini index of human wealth inequality increases over time.

Proposition 16 (consumption inequality). The Gini index of consumption is given by

$$
g_{c t}=\pi_{1}-\frac{\pi_{1} c_{1 t}}{\pi_{0} c_{0 t}+\pi_{1} c_{1 t}}=\pi_{1}-\frac{\pi_{1}}{\pi_{1}+\pi_{0} e^{\left(\rho_{1}-\rho_{0}\right) t}}
$$

The Gini index of consumption increases over time from 0 to $\pi_{1}$.

The dynamics of the aggregate consumption growth rate are complicated and depend on the regime considered. For simplicity, we focus on the more plausible situation. i.e. the case considered in subsection 4.2.1, whose dynamics are given Proposition 10. In this case, $\sigma_{10} \leq \sigma_{10}^{*}$ meaning that the more patient and talented class is relatively well endowed in human capital at the beginning.

Proposition 17 (consumption growth rate). In the case of Proposition 10, the dynamics of aggregate consumption and its growth rate are given by

$$
\begin{aligned}
c_{t} & =A H_{10} \frac{\rho(0)}{\sigma_{10}} \sum_{i=0}^{1} \frac{\pi_{i}}{B_{0}} e^{\left(B_{0}-\rho_{i}\right) t} \\
\gamma_{c t} & \equiv \frac{\dot{c}_{t}}{c_{t}}=\frac{\sum_{i=0}^{1} \pi_{i}\left(B_{0}-\rho_{i}\right) e^{\left(B_{0}-\rho_{i}\right) t}}{\sum_{i=0}^{1} \pi_{i} e^{\left(B_{0}-\rho_{i}\right) t}}
\end{aligned}
$$

Proposition 16 shows that, in the case of redistribution, the Gini index of consumption converges to $\pi_{1}$ or, equivalently, the consumption share of the impatient class converges to 0 . The same happens in the case of meritocracy (Proposition 5). However, in the case of meritocracy, the growth 
rate of consumption of the impatient class is $B_{1}-\rho_{1}$, while, in the case of redistribution considered in Proposition 17, it is equal to $B_{0}-\rho_{1}$. Thus, in this case of redistribution, the consumption of the impatient class grows at a higher rate.

We can now obtain the trade-off between inequality and growth.

Proposition 18 (trade-off inequality-growth). The (aggregate) consumption growth rate $\gamma_{c}$ is a function of the Gini index of consumption $g_{c}$. More precisely, the growth rate is an average whose weights depend on the Gini index:

$$
\gamma_{c}\left(g_{c}\right)=\sum_{i=0}^{1}\left(B_{0}-\rho_{i}\right) w_{i}\left(g_{c}\right)
$$

with

$$
w_{i}\left(g_{c}\right) \equiv \frac{\pi_{i}\left(\frac{\pi_{1}}{\pi_{0}} \frac{\pi_{0}+g_{c}}{\pi_{1}-g_{c}}\right)^{\frac{B_{0}-\rho_{i}}{\rho_{1}-\rho_{0}}}}{\sum_{j=0}^{1} \pi_{j}\left(\frac{\pi_{1}}{\pi_{0}} \frac{\pi_{0}+g_{c}}{\pi_{1}-g_{c}}\right)^{\frac{B_{0}-\rho_{j}}{\rho_{1}-\rho_{0}}}}
$$

$\gamma_{c}\left(g_{c}\right)$ is an increasing function, increasing from $\gamma_{c}(0)=B_{0}-\left(\pi_{0} \rho_{0}+\pi_{1} \rho_{1}\right)$ to $\gamma_{c}\left(\pi_{1}\right)=B_{0}-\rho_{0}$. Thus, the higher the social inequality, the higher the economic growth rate.

This proposition means that inequality promotes growth even in the case of redistribution. These results are consistent with empirical evidence. Many advanced economies have experienced increasing (income) inequality since the 1980s, see Atkinson (1999) and Goldin and Katz (2008). Moreover, according to Turnovski and Mitra (2013), this recent increase in inequality is explained by the increasing role of human capital as an engine of growth. ${ }^{7}$

\section{Discussion of the results and further com- ments}

Our first important result is that heterogeneity matters. Without heterogeneity, redistribution is not an issue and, therefore, the two policy regimes

\footnotetext{
${ }^{7}$ Goldin and Katz (1999, 2001) and Abramovitz and David (2000) find that the contribution of human capital to growth almost doubled during the 20th century in the United States, while the contribution of physical capital decreased.
} 
considered are indistinguishable. Also the effect of heterogeneity is much more pronounced in the case of redistribution. Indeed, in the meritocracy case the optimal solution for each agent is identical to the Lucas (1988) representative agent BGP, whereas with redistribution the optimal solution is never the BGP. The type of heterogeneity also plays an important role.

Although we did not discuss it previously, it is easy to see that, if we only had considered heterogeneity in the initial capital endowments, the optimal solution would converge to the Lucas BGP, being identical in the long run under both the meritocracy and redistribution regimes. When we introduce also different degrees of patience, the role of heterogeneity becomes more important and the optimal solutions of the two regimes are no longer asymptotically identical. However, they are both interior in what concerns working time. Finally, when we also consider heterogeneity in skills, in the redistribution case, the optimal solution for non-leisure time devoted to capital accumulation is never interior for both agents, being therefore dramatically different both from the meritocracy and from the representative agent solutions. A second important, although trivial result is that social welfare under the redistribution regime is always higher than under meritocracy. Indeed, the ability to redistribute cannot reduce overall welfare since one option available to the planner is to choose to not redistribute. Since we have seen that this is not the optimal path, the planner must do strictly better redistributing.

Together, these two results imply that it is optimal to exploit existing differences. We conjecture that, provided there is redistribution, welfare is higher (within mean preserving transformations) when we move from the representative agent case to an unequal distribution of skills. The proof is left for further works.

It is also interesting to note that, in the redistribution regime, the distribution of consumption is not affected by skills heterogeneity. Indeed only differences in patience, i.e. in preferences, are taken into account by the planner when she allocates consumption to each agent. However, skills differences are determinant for the allocation of tasks between agents. We found that, in the long run, the less talented class never invests in human capital, regardless of the initial distribution of human capital and of the efficiency of the less skilled agents in accumulating human capital. Also, whenever the human capital share of the more skilled class is below a certain critical threshold, these agents do not supply labor, devoting all their non-leisure time to capital accumulation at the beginning. We conclude that selection in the access 
to education and specialization are optimal.

We also find that, in both regimes, the inequality in the distribution of human capital increases in time. In the meritocracy regime this is due to differences in skills and in patience, which have therefore permanent effects, while with redistribution specialization is also an important driver of this result. Discussing now the relation between inequality and growth we find that in both regimes, in the long run, inequality promotes growth. It is easy to see that, within our framework, human capital accumulation is the channel through which inequality is associated with higher rates of growth. This happens, even with redistribution, suggesting that although redistribution increases utility and growth, it does not prevent a deterioration in the distribution of consumption during the growth process.

\section{Concluding remarks}

In this paper we extended the Lucas (1988) framework, introducing simultaneously heterogeneity in patience, in skills and in initial capital endowments. We considered two policy regimes: "meritocracy" and "redistribution". Our main conclusions are the following. First, heterogeneity changes significantly the optimal solution, specially in the presence of redistribution. Second, cooperation is always better, i.e. utility under the redistribution regime is always higher than under meritocracy. We conjecture that welfare is higher (within mean preserving transformations) when we move from a representative agent economy to an economy with an unequal distribution of skills. This means that it is optimal to exploit existing differences. Third, the redistribution of consumption only depends on preferences. In contrast, the distribution of tasks takes into account skills differences. Finally, we find that inequality is associated with higher rates of growth.

These results are novel, showing that heterogeneity really matters, which implies of course that the representative agent approach may be misleading. Another point of our contribution we want to stress, is that heterogeneity, instead of being considered a problem, should be optimally exploited in order to increase welfare. However, for this outcome redistribution is essential. In particular, we found that with redistribution, differences in skills should be translated into specialization in tasks and in a differential access to education. Note however that these results were obtained using a very stylized model. Therefore they should not be seen as policy recommendations. Nevertheless, 
in spite of the simplicity of the framework considered, the message that heterogeneity implies choices that are not only radically different from the ones associated with a representative agent economy, but which also dominate them in terms of welfare, should still be true in more general settings. Finally, in this work we have only considered the first best (planner's solution). Future work on the decentralized market solution is therefore welcome.

\section{Appendix}

Proof of Proposition 1. The Hamiltonian writes:

$$
\sum_{i=0}^{1} \pi_{i} e^{-\rho_{i} t} \ln \left(A h_{i t} u_{i t}\right)+\sum_{i=0}^{1} \lambda_{i t} h_{i t} B_{i}\left(1-u_{i t}\right)
$$

We have two state variables $\left(h_{i t}\right)$ and two controls $\left(u_{i t}\right)$.

We derive the first-order conditions: $\partial H_{t} / \partial \lambda_{i t}=\dot{h}_{i t}, \partial H_{t} / \partial h_{i t}=-\dot{\lambda}_{i t}$, $\partial H_{t} / \partial u_{i t}=0$ and the transversality condition $\lim _{t \rightarrow \infty}\left(\lambda_{i t} h_{i t}\right)=0$. The first-order conditions write

$$
\begin{aligned}
& \dot{u}_{i t}=\left(B_{i} u_{i t}-\rho_{i}\right) u_{i t} \\
& \dot{h}_{i t}=B_{i}\left(1-u_{i t}\right) h_{i t}
\end{aligned}
$$

jointly with the transversality condition $\lim _{t \rightarrow \infty}\left(e^{-\rho_{i} t} / u_{i t}\right)=0$.

Focus on the solution of ODE (60).

There are three cases.

(1) If $u_{i 0}<\rho_{i} / B_{i}$

$$
u_{i t}=\frac{1}{B_{i}} \frac{\rho_{i}}{1+e^{\rho_{i}\left(c_{i}+t\right)}}
$$

with

$$
c_{i}=\frac{1}{\rho_{i}} \ln \left(\frac{\rho_{i} / B_{i}}{u_{i 0}}-1\right)
$$

(2) If $u_{i 0}=\rho_{i} / B_{i}$

$$
u_{i t}=\frac{\rho_{i}}{B_{i}}
$$

(3) If $u_{i 0}>\rho_{i} / B_{i}$,

$$
\begin{aligned}
& u_{i t}=\frac{1}{B_{i}} \frac{\rho_{i}}{1-e^{\rho_{i}\left(c_{i}+t\right)}} \leq 1 \text { for } t \leq t^{*} \\
& u_{i t}=1 \text { for } t>t^{*}
\end{aligned}
$$


with

$$
\begin{aligned}
c_{i} & =\frac{1}{\rho_{i}} \ln \frac{B_{i} u_{i 0}-\rho_{i}}{B u_{i 0}}<0 \\
t^{*} & \equiv \frac{1}{\rho_{i}} \ln \left(1-\frac{\rho_{i}}{B_{i}}\right)-c_{i}>0
\end{aligned}
$$

(1) Focus on the case $u_{i 0}<\rho_{i} / B_{i}$.

We evaluate the utility along this trajectory:

$$
\int_{0}^{\infty} e^{-\rho_{i} t} \ln \left(A h_{i t} u_{i t}\right) d t=\frac{1}{\rho_{i}}\left[\ln \left(A h_{i 0} u_{i 0}\right)+\frac{B_{i}}{\rho_{i}}-1\right]
$$

(2) Consider the second case: $u_{i 0}=\rho_{i} / B_{i}$.

The economy is at the steady state from the beginning.

We evaluate the utility at the steady state.

$$
\int_{0}^{\infty} e^{-\rho_{i} t} \ln \left(A h_{i t} u_{i t}\right) d t=\frac{1}{\rho_{i}}\left[\ln \left(A h_{i 0} u_{i 0}\right)+\frac{B_{i}}{\rho_{i}}-1\right]
$$

Therefore, in the cases (1) and (2), we find:

$$
\begin{aligned}
& \arg \max _{u_{i 0} \leq \rho_{i} / B_{i}} \int_{0}^{\infty} e^{-\rho_{i} t} \ln \left(A h_{i t} u_{i t}\right) d t \\
= & \arg \max _{u_{i 0} \leq \rho_{i} / B_{i}} \frac{1}{\rho_{i}}\left[\ln \left(A h_{i 0} u_{i 0}\right)+\frac{B_{i}}{\rho_{i}}-1\right]=\frac{\rho_{i}}{B_{i}}
\end{aligned}
$$

and

$$
\max _{u_{i 0} \leq \rho_{i} / B_{i}} \int_{0}^{\infty} e^{-\rho_{i} t} \ln \left(A h_{i t} u_{i t}\right) d t=\frac{1}{\rho_{i}}\left[\ln \left(A h_{i 0} \frac{\rho_{i}}{B_{i}}\right)+\frac{B_{i}}{\rho_{i}}-1\right]
$$

(3) Focus on the case $u_{i 0}>\rho_{i} / B_{i}$.

The solution becomes

$$
\begin{aligned}
u_{i t} & =\frac{1}{B_{i}} \frac{\rho_{i}}{1-e^{\rho_{i}\left(c_{i}+t\right)}} \leq 1 \text { for } t \leq t^{*} \\
u_{i t} & =1 \text { for } t>t^{*}
\end{aligned}
$$

where

$$
\begin{aligned}
c_{i} & =\frac{1}{\rho_{i}} \ln \frac{B_{i} u_{i 0}-\rho_{i}}{B u_{i 0}}<0 \\
t^{*} & \equiv \frac{1}{\rho_{i}} \ln \left(1-\frac{\rho_{i}}{B_{i}}\right)-c_{i}>0
\end{aligned}
$$


We evaluate the utility along this trajectory.

$$
\begin{aligned}
& \int_{0}^{\infty} e^{-\rho_{i} t} \ln \left(A h_{i t} u_{i t}\right) d t=\int_{0}^{t^{*}} e^{-\rho_{i} t} \ln \left(A h_{i t} u_{i t}\right) d t+\int_{t^{*}}^{\infty} e^{-\rho_{i} t} \ln \left(A h_{i t^{*}}\right) d t \\
= & \frac{1}{\rho_{i}}\left[\frac{1-u_{i 0}}{u_{i 0}}+\ln \left(A h_{i 0} u_{i 0}\right)\right]
\end{aligned}
$$

Let us maximize

$$
\int_{0}^{\infty} e^{-\rho_{i} t} \ln \left(A h_{i t} u_{i t}\right) d t=\frac{1}{\rho_{i}}\left[\frac{1-u_{i 0}}{u_{i 0}}+\ln \left(A h_{i 0} u_{i 0}\right)\right]
$$

with respect to $u_{i 0}$. Notice that

$$
\frac{d}{d u_{i 0}}\left[\ln \left(A h_{i 0} u_{i 0}\right)+\frac{1-u_{i 0}}{u_{i 0}}\right]=\frac{1}{u_{i 0}}-\frac{1}{u_{i 0}^{2}}<0
$$

for $u_{i 0} \in\left[\rho_{i} / B_{i}, 1\right)$. Then, $\int_{0}^{\infty} e^{-\rho_{i} t} \ln \left(A h_{i t} u_{i t}\right) d t$ decreases in $\left[\rho_{i} / B_{i}, 1\right)$ and attains its maximum at $u_{i 0}^{*}=\rho_{i} / B_{i}$.

The value at $u_{i 0}^{*}=\rho_{i} / B_{i}$ is

$$
\int_{0}^{\infty} e^{-\rho_{i} t} \ln \left(A h_{i t} u_{i t}\right) d t=\frac{1}{\rho_{i}}\left(\ln \frac{A h_{i 0} \rho_{i}}{B_{i}}+\frac{B_{i}}{\rho_{i}}-1\right)
$$

We conclude that $u_{i 0}^{*}=\rho_{i} / B_{i}$ maximizes the utility whatever the case we consider. Since $u_{i 0}^{*}=\rho_{i} / B_{i}$ is also the steady state, we find that the BGP (9) is the planner's solution.

Proof of Proposition 2. Under the assumption $h_{0 t}>h_{1 t}$, the Gini index of wealth is given by:

$$
g_{h t}=\frac{\frac{\pi_{0} h_{0 t}+\pi_{1} h_{1 t}}{2}-\left(\frac{\pi_{1} \pi_{1} h_{1 t}}{2}+\pi_{0} \pi_{1} h_{1 t}+\frac{\pi_{0} \pi_{0} h_{0 t}}{2}\right)}{\frac{\pi_{0} h_{0 t}+\pi_{1} h_{1 t}}{2}}
$$

that is by (10).

Proof of Proposition 3. In order to compute the dynamics of the wealth Gini index, we consider the optimal individual wealth dynamics $(9): h_{i t}=h_{i 0} e^{\left(B_{i}-\rho_{i}\right) t}$.

Under Assumption 4, $B_{0}-\rho_{0}>B_{1}-\rho_{1}$. There are two cases: (1) $h_{00}>h_{10}$ and $(2) h_{00}<h_{10}$. 
(1) In the first case, $h_{0 t}>h_{1 t}$ for every $t$ and, then,

$$
g_{h t}=\pi_{1}-\frac{\pi_{1} h_{10} e^{\left(B_{1}-\rho_{1}\right) t}}{\pi_{0} h_{00} e^{\left(B_{0}-\rho_{0}\right) t}+\pi_{1} h_{10} e^{\left(B_{1}-\rho_{1}\right) t}}
$$

that is (11). It is easy to check that $g_{h t}$ increases in time.

(2) In the second case, $h_{00}<h_{10}$. Beyond the critical date $T_{h}$, the dominant consumer earns the higher revenue. Hence, $T_{h}$ is solution of $h_{0 T}=$ $h_{1 T}$, that is of $h_{00} e^{\left(B_{0}-\rho_{0}\right) T}=h_{10} e^{\left(B_{1}-\rho_{1}\right) T}$. We get (12).

Therefore, the Gini index of wealth is given by

$$
\begin{aligned}
& g_{h t}=\pi_{0}-\frac{\pi_{0} h_{0 t}}{\pi_{0} h_{0 t}+\pi_{1} h_{1 t}} \text { for } t \leq T_{h} \\
& g_{h t}=\pi_{1}-\frac{\pi_{1} h_{1 t}}{\pi_{0} h_{0 t}+\pi_{1} h_{1 t}} \text { for } t>T_{h}
\end{aligned}
$$

Replacing the expressions $h_{i t}=h_{i 0} e^{\left(B_{i}-\rho_{i}\right) t}$, we obtain (13) and (14).

Proof of Proposition 5. Under the assumption $c_{0 t}>c_{1 t}$, the Gini index of consumption is given by:

$$
g_{c t}=\frac{\frac{\pi_{0} c_{0 t}+\pi_{1} c_{1 t}}{2}-\left(\frac{\pi_{1} \pi_{1} c_{1 t}}{2}+\pi_{0} \pi_{1} c_{1 t}+\frac{\pi_{0} \pi_{0} c_{0 t}}{2}\right)}{\frac{\pi_{0} c_{0 t}+\pi_{1} c_{1 t}}{2}}
$$

that is by (15).

In order to compute the dynamics of the consumption Gini index, we consider the individual income dynamics: $c_{i t}=A h_{i t} u_{i t}=A h_{i 0} \rho_{i} e^{\left(B_{i}-\rho_{i}\right) t} / B_{i}=$ $c_{i 0} e^{\left(B_{i}-\rho_{i}\right) t}$.

Under Assumption 4, $B_{0}-\rho_{0}>B_{1}-\rho_{1}$. There are two cases: (1) $c_{00}>c_{10}$ and (2) $c_{00}<c_{10}$.

(1) In the first case, $c_{0 t}>c_{1 t}$ for every $t$ and, then,

$$
g_{c t}=\pi_{1}-\frac{\pi_{1} c_{10} e^{\left(B_{1}-\rho_{1}\right) t}}{\pi_{0} c_{00} e^{\left(B_{0}-\rho_{0}\right) t}+\pi_{1} c_{10} e^{\left(B_{1}-\rho_{1}\right) t}}
$$

that is (16). It is easy to check that $g_{c t}$ increases in time.

(2) In the second case, $c_{00}<c_{10}$. Beyond the critical date $T_{c}$, the dominant consumer earns the higher revenue. Hence, $T_{c}$ is solution of $c_{0 T}=c_{1 T}$, that is of $c_{00} e^{\left(B_{0}-\rho_{0}\right) T}=c_{10} e^{\left(B_{1}-\rho_{1}\right) T}$. We get $(17)$. 
Therefore, the Gini index is given by

$$
\begin{aligned}
& g_{c t}=\pi_{0}-\frac{\pi_{0} c_{0 t}}{\pi_{0} c_{0 t}+\pi_{1} c_{1 t}} \text { for } t \leq T_{c} \\
& g_{c t}=\pi_{1}-\frac{\pi_{1} c_{1 t}}{\pi_{0} c_{0 t}+\pi_{1} c_{1 t}} \text { for } t>T_{c}
\end{aligned}
$$

Replacing the expressions $c_{i t}=c_{i 0} e^{\left(B_{i}-\rho_{i}\right) t}$, we obtain (18) and (19).

Proof of Proposition 6. The aggregate consumption growth rate is given by

$$
\gamma_{t} \equiv \frac{\dot{c}_{t}}{c_{t}}=\frac{\left[\sum_{i=0}^{1} \pi_{i} c_{i 0} e^{\left(B_{i}-\rho_{i}\right) t}\right]^{\prime}}{\sum_{i=0}^{1} \pi_{i} c_{i 0} e^{\left(B_{i}-\rho_{i}\right) t}}=\frac{\sum_{i=0}^{1} \pi_{i} c_{i 0}\left(B_{i}-\rho_{i}\right) e^{\left(B_{i}-\rho_{i}\right) t}}{\sum_{i=0}^{1} \pi_{i} c_{i 0} e^{\left(B_{i}-\rho_{i}\right) t}}
$$

that is by $(20)$.

Proof of Proposition 7. Focus on case (1): $c_{00}>c_{10}$. From (16), we find

$$
e^{-\delta t}=\frac{\pi_{0} c_{00}}{\pi_{1} c_{10}} \frac{\pi_{1}-g_{t}}{\pi_{0}+g_{t}}
$$

Replacing this in (20), we obtain the trade-off (21) between inequality and growth.

Focus on case (2): $c_{00}<c_{10}$. In the case $t \leq T$, we have

$$
e^{-\delta t}=\frac{\pi_{0} c_{00}}{\pi_{1} c_{10}} \frac{\pi_{1}+g_{t}}{\pi_{0}-g_{t}}
$$

Replacing this in (20), we find the trade-off between the inequality and growth (22). Similarly, we obtain (23).

Proof of Proposition 8. In this case $B_{0}=B_{1}=B$, and $0<\rho_{0}<\rho_{1}$. By supposing that we have interior solution, the Hamiltonian writes:

$$
H_{t}=\left(\sum_{i=0}^{1} \pi_{i} e^{-\rho_{i} t}\right) \ln \sum_{i=0}^{1} \pi_{i} h_{i t} u_{i t}+B \sum_{i=0}^{1} \lambda_{i t}\left(1-u_{i t}\right) h_{i t}
$$

Since $h_{i t}$ maximizes $H_{t}, \partial H_{t} / \partial u_{i t}=0$, which implies:

$$
B \lambda_{i t}=\pi_{i} \frac{\sum_{j=0}^{1} \pi_{j} e^{-\rho_{j} t}}{\sum_{j=0}^{1} \pi_{j} h_{j t} u_{j t}}
$$


Replacing (61) in $\partial H_{t} / \partial h_{i t}=-\dot{\lambda}_{i t}$, we find

$$
-\dot{\lambda}_{i t}=\lambda_{i t} B u_{i t}+\lambda_{i t} B\left(1-u_{i t}\right)=B \lambda_{i t}
$$

Which implies $\lambda_{i t}=\lambda_{i 0} e^{-B t}$. We have also for any $t$,

$$
\frac{\lambda_{0 t}}{\lambda_{1 t}}=\frac{B \lambda_{0 t}}{B \lambda_{1 t}}=\frac{\pi_{0}}{\pi_{1}}
$$

Define

$$
\phi=\frac{\pi_{0}}{\lambda_{00}}=\frac{\pi_{1}}{\lambda_{10}}
$$

From (61), we get

$$
\sum_{i=0}^{1} \pi_{i} h_{i t} u_{i t}=\frac{\phi}{B} \sum_{i=0}^{1} \pi_{i} e^{\left(B-\rho_{i}\right) t}
$$

Define $H_{t} \equiv \sum_{i=0}^{1} H_{i t}=\sum_{i=0}^{1} \pi_{i} h_{i t}$. We have

$$
\dot{H}_{t}=\sum_{i=0}^{1} \pi_{t} \dot{h}_{i t}=\sum_{i=0}^{1} \pi_{i} B h_{i t}\left(1-u_{i t}\right)=B H_{t}-\phi \sum_{i=0}^{1} \pi_{i} e^{\left(B-\rho_{i}\right) t}
$$

Define

$$
z_{t}=H_{t}-\phi \sum_{i=0}^{1} \frac{\pi_{i}}{\rho_{i}} e^{\left(B-\rho_{i}\right) t}
$$

We get

$$
\begin{aligned}
\dot{z}_{t} & =\dot{H}_{t}-\phi \sum_{i=0}^{1} \frac{\pi_{i}}{\rho_{i}}\left(B-\rho_{i}\right) e^{\left(B-\rho_{i}\right) t} \\
& =B H_{t}-\phi \sum_{i=0}^{1} \pi_{t} e^{\left(B-\rho_{i}\right) t}-\phi \sum_{i=0}^{1} \frac{\pi_{i}}{\rho_{i}}\left(B-\rho_{i}\right) e^{\left(B-\rho_{i}\right) t} \\
& =B z_{t}
\end{aligned}
$$

This implies $z_{t}=z_{0} e^{B t}$ and

$$
H_{t}=z_{0} e^{B t}+\phi \sum_{i=0}^{1} \frac{\pi_{i}}{\rho_{i}} e^{\left(B-\rho_{i}\right) t}
$$


From the tranversality condition, we have

$$
\lim _{t \rightarrow \infty}\left(\lambda_{i t} h_{i t}\right)=\frac{\lambda_{i 0}}{\pi_{i}} \lim _{t \rightarrow \infty}\left(e^{-B t} \pi_{i} h_{i t}\right)=0
$$

Then, $\lim _{t \rightarrow \infty}\left(e^{-B t} \pi_{i} h_{i t}\right)=0$ and

$0=\lim _{t \rightarrow \infty}\left(e^{-B t} \sum_{i=0}^{1} \pi_{i} h_{i t}\right)=\lim _{t \rightarrow \infty}\left(e^{-B t} H_{t}\right)=\lim _{t \rightarrow \infty}\left(z_{0}+\phi \sum_{i=0}^{1} \frac{\pi_{i}}{\rho_{i}} e^{-\rho_{i} t}\right)=z_{0}$

which implies $z_{t}=0$ for any $t$. Thus,

$$
\sum_{i=0}^{1} \pi_{i} h_{i t}=H_{t}=\phi \sum_{i=0}^{1} \frac{\pi_{i}}{\rho_{i}} e^{\left(B-\rho_{i}\right) t}
$$

We can guess that the solution must satisfies, for $i=0,1$, equation (26) where $\zeta_{0}$ and $\zeta_{1}$ satisfy $\sum_{i=0}^{1} \pi_{i} \zeta_{i}=1$. We observe that $\zeta_{i}$ in (27) satisfies this condition.

We can also guess that

$$
h_{i t} u_{i t}=\frac{\phi}{B} \zeta_{i} \sum_{j=0}^{1} \pi_{j} e^{\left(B-\rho_{j}\right) t}
$$

We observe that (63) satisfies (26).

This is equivalent to

$$
u_{i t}=\frac{\frac{\phi}{B} \zeta_{i} \sum_{j=0}^{1} \pi_{j} e^{\left(B-\rho_{j}\right) t}}{\phi \zeta_{i} \sum_{j=0}^{1} \frac{\pi_{j}}{\rho_{j}} e^{\left(B-\rho_{j}\right) t}}
$$

that is $(26)$.

We can now construct the solution. Considering (62) with $t=0$, we get $\phi$ in $(26)$.

Considering (63) with $t=0$, we get

$$
\zeta_{i}=\frac{h_{i 0}}{\phi \sum_{i=0}^{1} \pi_{j} / \rho_{j}}
$$

that is $\zeta_{i}$ in (26). We observe that $\sum_{i=0}^{1} \pi_{i} \zeta_{i}=1$. 
Define $\lambda_{i 0}=\pi_{i} / \phi$ yielding $\lambda_{i t}=e^{-B t} \pi_{i} / \phi$.

Observe that $0<u_{i t}<1$ and $\dot{h}_{i t}=B h_{i t}-B h_{i t} u_{i t}=B h_{i t}\left(1-u_{i t}\right)$.

We can verify easily that $\left(h_{i t}, u_{i t}, \lambda_{i t}\right)$ satisfies Pontryagin conditions.

Observe that in this case $\left(B_{0}=B_{1}=B\right)$, we have $u_{0 t}=u_{1 t}=u_{t}$, which converges to $\rho_{0} / B$. The total human capital stock $H_{t}=\sum_{i=0}^{1} \pi_{i} h_{i t}$, satisfies $\dot{H}_{t}=B H_{t}\left(1-u_{t}\right)$.

Proof of Lemma 9. Suppose the contrary: there exists $t$ such that, for every $i, 0<u_{i t}<1$. This implies the existence of $t_{1}<t_{2}$ such that $0<u_{i t}<1$ for every $t_{1}<t<t_{2}$. On the interval $\left(t_{1}, t_{2}\right)$ the Hamiltonian writes:

$$
H_{t}=\left(\sum_{i=0}^{1} \pi_{i} e^{-\rho_{i} t}\right) \ln \sum_{i=0}^{1} \pi_{i} h_{i t} u_{i t}+\sum_{i=0}^{1} \lambda_{i t} B_{i}\left(1-u_{i t}\right) h_{i t}
$$

For every $t_{1}<t<t_{2}$, we have $\partial H_{t} / \partial u_{i t}=0, \partial H_{t} / \partial \lambda_{i t}=\dot{h}_{i t}$ and $\partial H_{t} / \partial h_{i t}=-\dot{\lambda}_{i t}$. The first equation implies that, for every $i$ :

$$
B_{i} \lambda_{i t}=\pi_{i} \frac{\sum_{j=0}^{1} \pi_{j} e^{-\rho_{j} t}}{\sum_{j=0}^{1} \pi_{j} h_{j t} u_{j t}}
$$

Replacing (64) in $\partial H_{t} / \partial h_{i t}=-\dot{\lambda}_{i t}$, we find

$$
-\dot{\lambda}_{i t}=\lambda_{i t} B_{i} u_{i t}+\lambda_{i t} B_{i}\left(1-u_{i t}\right)=\lambda_{i t} B_{i}
$$

Integrating from $t_{1}$ to $t$, we obtain $\lambda_{i t}=\lambda_{i t_{1}} e^{-B_{i}\left(t-t_{1}\right)}$ for any $t_{1}<t<$ $t_{2}$. From (64), we have also $B_{0} \lambda_{0 t} /\left(B_{1} \lambda_{1 t}\right)=\pi_{0} / \pi_{1}$. This implies that $e^{-\left(B_{0}-B_{1}\right)\left(t-t_{1}\right)}=\pi_{0} B_{1} \lambda_{1 t_{1}} /\left(\pi_{1} B_{0} \lambda_{0 t_{1}}\right)$ for any $t_{1}<t<t_{2}$. This leads to a contradiction with $B_{0} \neq B_{1}$ since, in this case, the left-hand side changes over time.

Proof of Proposition 10. The Hamiltonian writes

$$
H_{t}=\left(\sum_{i=0}^{1} \pi_{i} e^{-\rho_{i} t}\right) \ln \sum_{i=0}^{1} \pi_{i} h_{i t} u_{i t}+\sum_{i=0}^{1} \lambda_{i t} B_{i}\left(1-u_{i t}\right) h_{i t}
$$

Lemma 9 allows us to focus on a candidate solution which is a corner solution for one agent: there exists $T \geq 0$ such that

$$
0<u_{0 t}<1 \text { and } u_{1 t}=1
$$


for every $t \geq T$. We will show that $T=0$ and the Pontryagin's optimal conditions are satisfied.

If (65) is solution, we have

$$
\begin{aligned}
\left.\frac{\partial H_{t}}{\partial u_{0 t}}\right|_{0<u_{0 t}<1} & =\pi_{0} h_{0 t} \frac{\sum_{i=0}^{1} \pi_{i} e^{-\rho_{i} t}}{\sum_{i=0}^{1} \pi_{i} h_{i t} u_{i t}}-B_{0} \lambda_{0 t} h_{0 t}=0 \\
\left.\frac{\partial H_{t}}{\partial u_{1 t}}\right|_{u_{1 t}=1} & =\pi_{1} h_{1 t} \frac{\sum_{i=0}^{1} \pi_{i} e^{-\rho_{i} t}}{\sum_{i=0}^{1} \pi_{i} h_{i t} u_{i t}}-B_{1} \lambda_{1 t} h_{1 t} \geq 0
\end{aligned}
$$

Therefore,

$$
\begin{aligned}
& B_{0} \lambda_{0 t}=\pi_{0} \frac{\sum_{i=0}^{1} \pi_{i} e^{-\rho_{i} t}}{\sum_{i=0}^{1} \pi_{i} h_{i t} u_{i t}} \\
& 0<u_{0 t}<1 \text { implies } \\
& \dot{\lambda}_{0 t}=-\frac{\partial H_{t}}{\partial h_{0 t}}=-\pi_{0} u_{0 t} \frac{\sum_{i=0}^{1} \pi_{i} e^{-\rho_{i} t}}{\sum_{i=0}^{1} \pi_{i} h_{i t} u_{i t}}-B_{0} \lambda_{0 t}\left(1-u_{0 t}\right)=-B_{0} \lambda_{0 t}
\end{aligned}
$$

(69) entails

$$
\lambda_{0 t}=\lambda_{0 T} e^{-B_{0}(t-T)}
$$

for any $t \geq T$.

$u_{1 t}=1$ implies

$$
\dot{\lambda}_{1 t}=-\frac{\partial H_{t}}{\partial h_{1 t}}=-\pi_{1} u_{1 t} \frac{\sum_{i=0}^{1} \pi_{i} e^{-\rho_{i} t}}{\sum_{i=0}^{1} \pi_{i} h_{i t} u_{i t}}-B_{1} \lambda_{1 t}\left(1-u_{1 t}\right)=-\frac{\pi_{1}}{\pi_{0}} B_{0} \lambda_{0 t}
$$

Moreover,

$$
\dot{\lambda}_{1 t}=-\frac{\pi_{1}}{\pi_{0}} B_{0} \lambda_{0 t}=\frac{\pi_{1}}{\pi_{0}} \dot{\lambda}_{0 t}
$$

that is

$$
\lambda_{1 t}=\frac{\pi_{1}}{\pi_{0}}\left(\lambda_{0 t}-\lambda_{0 T}\right)+\lambda_{1 T}=\frac{\pi_{1}}{\pi_{0}} \lambda_{0 T} e^{-B_{0}(t-T)}+\lambda_{1 T}-\frac{\pi_{1}}{\pi_{0}} \lambda_{0 T}
$$

A candidate solution satisfies the transversality condition, that is

$$
\lim _{t \rightarrow \infty}\left(\lambda_{1 t} h_{1 t}\right)=\lim _{t \rightarrow \infty}\left[\frac{\pi_{1}}{\pi_{0}} \lambda_{0 T} e^{-B_{0}(t-T)} h_{1 t}\right]+\lim _{t \rightarrow \infty}\left[\left(\lambda_{1 T}-\frac{\pi_{1}}{\pi_{0}} \lambda_{0 T}\right) h_{1 t}\right]=0
$$


which holds only if $\lambda_{1 T}-\lambda_{0 T} \pi_{1} / \pi_{0}=0$. According to (71), we find

$$
\lambda_{1 t}=\frac{\pi_{1}}{\pi_{0}} \lambda_{0 t}
$$

Replacing (70) in equation (68), we get

$$
\sum_{i=0}^{1} \pi_{i} h_{i t} u_{i t}=\frac{\pi_{0}}{B_{0} \lambda_{0 T} e^{B_{0} T}} \sum_{i=0}^{1} \pi_{i} e^{\left(B_{0}-\rho_{i}\right) t}
$$

and, noticing that $u_{1 t}=1$ and $h_{1 t}=h_{1 T}$ for every $t \geq T$, we obtain

$$
h_{0 t} u_{0 t}=\frac{1}{B_{0} \lambda_{0 T} e^{B_{0} T}} \sum_{i=0}^{1} \pi_{i} e^{\left(B_{0}-\rho_{i}\right) t}-\frac{\pi_{1}}{\pi_{0}} h_{1 T}
$$

Therefore,

$$
\dot{h}_{0 t}=B_{0} h_{0 t}-B_{0} u_{0 t} h_{0 t}=B_{0} h_{0 t}+B_{0} \frac{\pi_{1}}{\pi_{0}} h_{1 T}-\frac{\sum_{i=0}^{1} \pi_{i} e^{\left(B_{0}-\rho_{i}\right) t}}{\lambda_{0 T} e^{B_{0} T}}
$$

This differential equation is equivalent to $\dot{z}_{t}=B_{0} z_{t}$, where

$$
z_{t} \equiv h_{0 t}+\frac{\pi_{1}}{\pi_{0}} h_{1 T}-\frac{\sum_{i=0}^{1} \frac{\pi_{i}}{\rho_{i}} e^{\left(B_{0}-\rho_{i}\right) t}}{\lambda_{0 T} e^{B_{0} T}}
$$

The solution of $\dot{z}_{t}=B_{0} z_{t}$ is $z_{t}=z_{T} e^{B_{0}(t-T)}$ for $t \geq T$. Thus

$$
h_{0 t}=z_{T} e^{B_{0}(t-T)}-\frac{\pi_{1}}{\pi_{0}} h_{1 T}+\frac{\sum_{i=0}^{1} \frac{\pi_{i}}{\rho_{i}} e^{\left(B_{0}-\rho_{i}\right) t}}{\lambda_{0 T} e^{B_{0} T}}
$$

A candidate solution satisfies the transversality condition, that is, according to (70),

$$
\lim _{t \rightarrow \infty}\left(\lambda_{0 t} h_{0 t}\right)=\lim _{t \rightarrow \infty}\left(\lambda_{0 T} z_{T}\right)+\lim _{t \rightarrow \infty}\left(\lambda_{0 t}\left[\frac{\sum_{i=0}^{1} \frac{\pi_{i}}{\rho_{i}} e^{\left(B_{0}-\rho_{i}\right) t}}{\lambda_{0 T} e^{B_{0} T}}-\frac{\pi_{1}}{\pi_{0}} h_{1 T}\right]\right)=0
$$

which holds only if $z_{T}=0$, that is only if

$$
\pi_{1} h_{1 T}+\pi_{0} h_{0 T}=\pi_{0} \frac{\sum_{i=0}^{1} \frac{\pi_{i}}{\rho_{i}} e^{\left(B_{0}-\rho_{i}\right) T}}{\lambda_{0 T} e^{B_{0} T}}=\frac{\pi_{0}}{\lambda_{0 T}} \sum_{i=0}^{1} \frac{\pi_{i}}{\rho_{i}} e^{-\rho_{i} T}
$$


(68) and (74) imply

$$
\frac{B_{0}}{\rho(T)}=\frac{\pi_{0} h_{0 T}+\pi_{1} h_{1 T}}{\pi_{1} h_{1 T}+\pi_{0} h_{0 T} u_{0 T}}
$$

where $\rho(T)$ is given by $(28)$.

We compute $u_{0 T}$ from (75) and we impose the economic restriction $0 \leq$ $u_{0 T} \leq 1$ or, equivalently,

$$
1 \leq \frac{B_{0}}{\rho(T)} \leq \frac{1}{\sigma_{1 T}}
$$

( $0 \leq u_{0 T}$ is equivalent to the RHS, while $u_{0 T} \leq 1$ to the LHS)

Inequality in the LHS of (76) is satisfied by Assumption 5: $B_{0}>\rho_{1}>$ $\rho(T)$.

Thus, the candidate solution holds for every $t \geq 0$ only if the RHS holds at $T=0$. It is the case because (76) with $T=0$ is equivalent to the initial condition $\sigma_{10} \leq \sigma_{10}^{*}$.

Let us now provide the explicit trajectory for $h_{i t}$ and $u_{i t}$ and show that the optimal conditions are verified.

From (75) with $T=0$, we have

$$
u_{00}=\frac{\rho(0)}{B_{0}}-\left[1-\frac{\rho(0)}{B_{0}}\right] \frac{H_{10}}{H_{00}}
$$

As seen above, $0 \leq u_{00} \leq 1$ under Assumption 5 and inequality $\sigma_{10} \leq$ $\rho(0) / B_{0}$.

From (68) and (74) with $T=0$, we find $\lambda_{00}$.

Moreover, (73) with $z_{T}=0$ and $T=0$ implies

$$
h_{0 t}=\frac{1}{\lambda_{00}} \sum_{i=0}^{1} \frac{\pi_{i}}{\rho_{i}} e^{\left(B_{0}-\rho_{i}\right) t}-\frac{\pi_{1}}{\pi_{0}} h_{10}
$$

Replacing (77) in (72) with $T=0$ and solving for $u_{0 t}$, we get (31).

Let us show that

$$
\begin{aligned}
\lambda_{0 t} & =\frac{\pi_{0} e^{-B_{0} t}}{H_{00}+H_{10}} \sum_{i=0}^{1} \frac{\pi_{i}}{\rho_{i}} \\
\lambda_{1 t} & =\frac{\pi_{1}}{\pi_{0}} \lambda_{0 t}
\end{aligned}
$$


and (32) to (31) satisfy the Maximum Principle by Pontryagin.

First, replacing (78), (30) and (31) in (66), we find

$$
\left.\frac{\partial H_{t}}{\partial u_{0 t}}\right|_{0<u_{0 t}<1}=0
$$

Assumption $5\left(B_{0}>B_{1}\right)$ implies

$$
B_{1} \lambda_{1 t}<B_{0} \lambda_{1 t}=B_{0} \lambda_{0 t} \frac{\pi_{1}}{\pi_{0}}=\frac{\pi_{1} \sum_{i=0}^{1} \pi_{i} e^{-\rho_{i} t}}{\pi_{0} h_{0 t} u_{0 t}+\pi_{1} h_{10}}
$$

and, so, according to (67), also

$$
\left.\frac{\partial H_{t}}{\partial u_{1 t}}\right|_{u_{1 t}=1} \geq 0
$$

Moreover,

$$
\begin{aligned}
& \dot{\lambda}_{0 t}=-\lambda_{0 t} B_{0}=-\frac{\partial H_{t}}{\partial h_{0 t}} \\
& \dot{\lambda}_{1 t}=-\frac{\pi_{1} \sum_{i} \pi_{i} e^{-\rho_{i} t}}{\pi_{1} h_{10}+\pi_{0} h_{0 t} u_{0 t}}=-\frac{\pi_{1}}{\pi_{0}} \lambda_{0 t} B_{0}=-\frac{\partial H_{t}}{\partial h_{1 t}}
\end{aligned}
$$

Finally, we check the transversality conditions. Since

$$
\begin{aligned}
\lim _{t \rightarrow \infty}\left(\lambda_{i t} h_{i t}\right) & =\lim _{t \rightarrow \infty}\left(\lambda_{i 0} e^{-B_{i} t} h_{i t}\right)=\lambda_{i 0} \lim _{t \rightarrow \infty}\left(e^{-B_{i} t} h_{i t}\right) \\
& =\lambda_{i 0} \lim _{t \rightarrow \infty}\left[e^{-B_{i} t} h_{i 0} e^{\int_{0}^{t} B_{i}\left(1-u_{i s}\right) d s}\right]=\lambda_{i 0} e^{-B_{i} \int_{0}^{\infty} u_{i s} d s}
\end{aligned}
$$

the transversality conditions are equivalent to $\int_{0}^{\infty} u_{i s} d s=\infty$. These equalities are satisfied because $\lim _{t \rightarrow \infty} u_{0 t}=\rho_{0} / B_{0}>0$ and $u_{1 t}=1$ for every $t$.

Proof of Proposition 12. (1) Focus first on the case $B_{1} \leq B_{1}^{*}$.

Lemma 9 allows us to focus on a candidate solution which is a corner solution for one agent. We prove the first subcase by considering a potential solution such that

$$
\begin{aligned}
u_{0 t} & =0 \text { for } 0 \leq t \leq T \\
0<u_{0 t} & <1 \text { for } t>T \\
u_{1 t} & =1 \text { for any } t
\end{aligned}
$$


by deriving its explicit trajectory and checking that this trajectory satisfies the Pontryagin's Maximum Principle.

According to the Maximum Principle, we need

$$
\begin{aligned}
& \left.\frac{\partial H_{t}}{\partial u_{0 t}}\right|_{u_{0 t}=0}=\pi_{0} h_{0 t} \frac{\sum_{i=0}^{1} \pi_{i} e^{-\rho_{i} t}}{\sum_{i=0}^{1} \pi_{i} h_{i t} u_{i t}}-B_{0} \lambda_{0 t} h_{0 t} \leq 0 \\
& \left.\frac{\partial H_{t}}{\partial u_{1 t}}\right|_{u_{1 t}=1}=\pi_{1} h_{1 t} \frac{\sum_{i=0}^{1} \pi_{i} e^{-\rho_{i} t}}{\sum_{i=0}^{1} \pi_{i} h_{i t} u_{i t}}-B_{1} \lambda_{1 t} h_{1 t} \geq 0
\end{aligned}
$$

for any $t \in[0, T]$, that is

$$
B_{0} \lambda_{0 t} \geq \frac{\pi_{0}}{\pi_{1}} \frac{\sum_{i=0}^{1} \pi_{i} e^{-\rho_{i} t}}{h_{10}} \text { and } B_{1} \lambda_{1 t} \leq \frac{\sum_{i=0}^{1} \pi_{i} e^{-\rho_{i} t}}{h_{10}}
$$

Moreover, for any $t \in[0, T]$,

$$
\begin{aligned}
& \dot{\lambda}_{0 t}=-\frac{\partial H_{t}}{\partial h_{0 t}}=-\pi_{0} u_{0 t} \frac{\sum_{i=0}^{1} \pi_{i} e^{-\rho_{i} t}}{\sum_{i=0}^{1} \pi_{i} h_{i t} u_{i t}}-B_{0} \lambda_{0 t}\left(1-u_{0 t}\right) \\
& \dot{\lambda}_{1 t}=-\frac{\partial H_{t}}{\partial h_{1 t}}=-\pi_{1} u_{1 t} \frac{\sum_{i=0}^{1} \pi_{i} e^{-\rho_{i} t}}{\sum_{i=0}^{1} \pi_{i} h_{i t} u_{i t}}-B_{1} \lambda_{1 t}\left(1-u_{1 t}\right)
\end{aligned}
$$

that is

$$
\dot{\lambda}_{0 t}=-B_{0} \lambda_{0 t} \text { and } \dot{\lambda}_{1 t}=-\frac{\sum_{i=0}^{1} \pi_{i} e^{-\rho_{i} t}}{h_{10}}
$$

with solutions

$$
\lambda_{0 t}=\lambda_{00} e^{-B_{0} t} \text { and } \lambda_{1 t}=\lambda_{10}-\frac{1}{h_{10}} \sum_{i=0}^{1} \frac{\pi_{i}}{\rho_{i}}\left(1-e^{-t \rho_{i}}\right)
$$

If $t>T$, according to the Maximum Principle, we require

$$
\left.\frac{\partial H_{t}}{\partial u_{0 t}}\right|_{0<u_{0 t}<1}=\pi_{0} h_{0 t} \frac{\sum_{i=0}^{1} \pi_{i} e^{-\rho_{i} t}}{\sum_{i=0}^{1} \pi_{i} h_{i t} u_{i t}}-B_{0} \lambda_{0 t} h_{0 t}=0
$$

that is

$$
B_{0} \lambda_{0 t}=\pi_{0} \frac{\sum_{i=0}^{1} \pi_{i} e^{-\rho_{i} t}}{\sum_{i=0}^{1} \pi_{i} h_{i t} u_{i t}}
$$


which implies in turn

$$
\dot{\lambda}_{0 t}=-u_{0 t}\left(\pi_{0} \frac{\sum_{i=0}^{1} \pi_{i} e^{-\rho_{i} t}}{\sum_{i=0}^{1} \pi_{i} h_{i t} u_{i t}}\right)-B_{0} \lambda_{0 t}\left(1-u_{0 t}\right)=-B_{0} \lambda_{0 t}
$$

with explicit solution $\lambda_{0 t}=\lambda_{00} e^{-B_{1} t}$. Thus, this trajectory holds for every $t$.

If $0 \leq t \leq T$, we obtain $\dot{h}_{0 t}=B_{0} h_{0 t}$ from $\dot{h}_{0 t}=B_{0}\left(1-u_{0 t}\right) h_{0 t}$, that is $h_{0 t}=h_{00} e^{B_{0} t}$.

If $t \geq T,(83)$ and $u_{1 t}=1$ entail

$$
\dot{\lambda}_{1 t}=-\pi_{1} u_{1 t} \frac{\sum_{i=0}^{1} \pi_{i} e^{-\rho_{i} t}}{\sum_{i=0}^{1} \pi_{i} h_{i t} u_{i t}}-B_{1} \lambda_{1 t}\left(1-u_{1 t}\right)=-\frac{\pi_{1}}{\pi_{0}} B_{0} \lambda_{0 t}=\frac{\pi_{1}}{\pi_{0}} \dot{\lambda}_{0 t}
$$

and

$$
\lambda_{1 t}=\frac{\pi_{1}}{\pi_{0}}\left(\lambda_{0 t}-\lambda_{0 T}\right)+\lambda_{1 T}=\frac{\pi_{1}}{\pi_{0}} \lambda_{0 T} e^{-B_{0}(t-T)}+\lambda_{1 T}-\frac{\pi_{1}}{\pi_{0}} \lambda_{0 T}
$$

According to the Maximum Principle, a candidate trajectory satisfies also the transversality condition:

$$
\lim _{t \rightarrow \infty}\left(\lambda_{1 t} h_{1 t}\right)=\lim _{t \rightarrow \infty}\left[\frac{\pi_{1}}{\pi_{0}} \lambda_{0 T} e^{-B_{0}(t-T)} h_{1 t}\right]+\lim _{t \rightarrow \infty}\left[\left(\lambda_{1 T}-\frac{\pi_{1}}{\pi_{2}} \lambda_{2 T}\right) h_{1 t}\right]=0
$$

which holds only if $\lambda_{1 T}-\lambda_{0 T} \pi_{1} / \pi_{0}=0$. Therefore, (84) implies $\lambda_{1 t}=$ $\lambda_{0 t} \pi_{1} / \pi_{0}$, for any $t \geq T$. In particular, we get $\lambda_{1 T}=\lambda_{0 T} \pi_{1} / \pi_{0}$. Replacing $\lambda_{0 t}=\lambda_{00} e^{-B_{0} t}$ in equation (83), we get

$$
\sum_{i=0}^{1} \pi_{i} h_{i t} u_{i t}=\frac{\pi_{0} \sum_{i=0}^{1} \pi_{i} e^{-\rho_{i} t}}{B_{0} \lambda_{00} e^{-B_{0} t}}=\frac{\pi_{0} \sum_{i=0}^{1} \pi_{i} e^{\left(B_{0}-\rho_{i}\right) t}}{B_{0} \lambda_{0 T} e^{B_{0} T}}
$$

and, noticing that $u_{1 t}=1$ and $h_{1 t}=h_{1 T}$ for every $t \geq T$, we obtain

$$
h_{0 t} u_{0 t}=\frac{\sum_{i=0}^{1} \pi_{i} e^{\left(B_{0}-\rho_{i}\right) t}}{B_{0} \lambda_{0 T} e^{B_{0} T}}-\frac{\pi_{1}}{\pi_{0}} h_{1 T}
$$

Therefore, for $t \geq T$,

$$
\dot{h}_{0 t}=B_{0} h_{0 t}-B_{0} h_{0 t} u_{0 t}=B_{0} h_{0 t}+B_{0} \frac{\pi_{1}}{\pi_{0}} h_{1 T}-\frac{\sum_{i=0}^{1} \pi_{i} e^{\left(B_{0}-\rho_{i}\right) t}}{\lambda_{0 T} e^{B_{0} T}}
$$


or, equivalently, $\dot{z}_{t}=B_{0} z_{t}$ with

$$
z_{t} \equiv h_{0 t}+\frac{\pi_{1}}{\pi_{0}} h_{1 T}-\frac{\sum_{i=0}^{1} \frac{\pi_{i}}{\rho_{i}} e^{\left(B_{0}-\rho_{i}\right) t}}{\lambda_{0 T} e^{B_{0} T}}
$$

whose solution is $z_{t}=z_{T} e^{B_{0}(t-T)}$. Thus,

$$
h_{0 t}=z_{T} e^{B_{0}(t-T)}-\frac{\pi_{1}}{\pi_{0}} h_{1 T}+\frac{\sum_{i=0}^{1} \frac{\pi_{i}}{\rho_{i}} e^{\left(B_{0}-\rho_{i}\right) t}}{\lambda_{0 T} e^{B_{0} T}}
$$

for $t \geq T$.

As above, a candidate solution satisfies also the transversality condition:

$$
\lim _{t \rightarrow \infty}\left(\lambda_{0 t} h_{0 t}\right)=\lim _{t \rightarrow \infty}\left(\lambda_{0 T} z_{T}\right)+\lim _{t \rightarrow \infty}\left(\lambda_{0 t}\left[\frac{\sum_{i=0}^{1} \frac{\pi_{i}}{\rho_{i}} e^{\left(B_{0}-\rho_{i}\right) t}}{\lambda_{0 T} e^{B_{0} T}}-\frac{\pi_{1}}{\pi_{0}} h_{1 T}\right]\right)=0
$$

which holds only if $z_{T}=0$ or, equivalently,

$$
\pi_{1} h_{1 T}+\pi_{0} h_{0 T}=\pi_{0} \frac{\sum_{i=0}^{1} \frac{\pi_{i}}{\rho_{i}} e^{\left(B_{0}-\rho_{i}\right) T}}{\lambda_{0 T} e^{B_{0} T}}=\frac{\pi_{0}}{\lambda_{0 T}} \sum_{i=0}^{1} \frac{\pi_{i}}{\rho_{i}} e^{-\rho_{i} T}
$$

(83) and (85) imply

$$
\frac{B_{0}}{\rho(T)}=\frac{\pi_{0} h_{0 T}+\pi_{1} h_{1 T}}{\pi_{0} h_{0 T} u_{0 T}+\pi_{1} h_{1 T}}
$$

Since $u_{0 T}=0, h_{1 T}=h_{10}, h_{0 T}=h_{00} e^{B_{0} T}$ and $H_{i 0} \equiv \pi_{i} h_{i 0},(86)$ is equivalent to $(35)$.

The critical time $T$ of the candidate trajectory (80) is precisely the solution $T_{0}$ of equation (35). This solution exists and is positive. Indeed, we know that $\rho(T) \in\left[\rho_{0}, \rho(0)\right]$, a bounded interval. Moreover, (35) is equivalent to $e^{B_{0} T} H_{00} / H_{10}=B_{0} / \rho(T)-1$, whose RHS is positive according to Assumption $5\left(B_{0}>\rho_{1}>\rho(T)\right)$ and bounded from above. The LHS goes from $H_{00} / H_{10}$ to $\infty$. If $H_{00} / H_{10}<B_{0} / \max \rho(T)-1=B_{0} / \rho(0)-1$, then a solution $T_{0}$ exists. But this inequality is precisely equivalent to inequality $\sigma_{10}>\sigma_{10}^{*}$, precisely the case we are considering in Proposition (12).

From (85), we get

$$
\lambda_{0 T_{0}}=\frac{\pi_{0} \sum_{i=0}^{1} \frac{\pi_{i}}{\rho_{i}} e^{-\rho_{i} T_{0}}}{H_{00} e^{B_{0} T_{0}}+H_{10}}
$$


and, from (35),

$$
\lambda_{0 T_{0}}=\frac{\pi_{0} \sum_{i=0}^{1} \pi_{i} e^{-\rho_{i} T_{0}}}{B_{0} H_{10}}
$$

We want to prove that the trajectory defined by equations

$$
\begin{aligned}
& \lambda_{0 t}=\lambda_{0 T_{0}} e^{B_{0}\left(T_{0}-t\right)} \text { for any } t \\
& \lambda_{1 t}=\frac{\pi_{1}}{\pi_{0}} \lambda_{0 T_{0}}+\frac{1}{h_{10}} \sum_{i=0}^{1} \frac{\pi_{i}}{\rho_{i}}\left(e^{-\rho_{i} t}-e^{-\rho_{i} T_{0}}\right) \text { for } 0 \leq t \leq T_{0} \\
& \lambda_{1 t}=\frac{\pi_{1}}{\pi_{0}} \lambda_{0 T_{0}} e^{B_{0}\left(T_{0}-t\right)} \text { for } t>T_{0}
\end{aligned}
$$

and (44) to (43) satisfies the Pontryagin's Maximum Principle.

It is easy to check that $\lambda_{0 t}$ and $\lambda_{1 t}$ are differentiable functions with respect to $t$ in $[0,+\infty)$ with $\dot{\lambda}_{i t}=-\partial H_{t} / \partial h_{i t}$ and that, for any $t$, we have $\dot{h}_{0 t}=$ $B_{0}\left(1-u_{0 t}\right) h_{0 t}$.

Let us show that $u_{0 t}$ and $u_{1 t}$ maximize the Hamiltonian. For $t>T_{0}$, apply the proof of Proposition 10. Focus now on $0 \leq t \leq T_{0}$.

We want to prove that $\partial H_{t} /\left.\partial u_{0 t}\right|_{u_{0 t}=0} \leq 0$.

Since $\sum_{i=0}^{1} \pi_{i} e^{\left(B_{0}-\rho_{i}\right) t}$ is strictly increasing and

$$
\frac{B_{0} \sum_{i=0}^{1} \frac{\pi_{i}}{\rho_{i}} e^{\left(B_{0}-\rho_{i}\right) T_{0}}}{1+e^{B_{0} T_{0}} H_{00} / H_{10}}=\sum_{i=0}^{1} \pi_{i} e^{\left(B_{0}-\rho_{i}\right) T_{0}}
$$

then, for any $t \in\left[0, T_{0}\right]$, we have

$$
\frac{B_{0} \sum_{i=0}^{1} \frac{\pi_{i}}{\rho_{i}} e^{\left(B_{0}-\rho_{i}\right) T_{0}}}{1+e^{B_{0} T_{0}} H_{00} / H_{10}} \geq \sum_{i=0}^{1} \pi_{i} e^{\left(B_{0}-\rho_{i}\right) T_{0}}
$$

or, equivalently,

$$
B_{0} \lambda_{0 t}=B_{0} \lambda_{0 T_{0}} e^{B_{0}\left(T_{0}-t\right)} \geq \pi_{0} \frac{\sum_{i=0}^{1} \pi_{i} e^{-\rho_{i} t}}{\sum_{i=0}^{1} \pi_{i} h_{i t} u_{i t}}
$$

because $u_{0 t}=0$ for $0 \leq t \leq T_{0}$ and $u_{1 t}=1$ for any $t$. Then, (81) is verified. This means that $u_{0 t}=0$ maximizes the Hamiltonian.

We want to prove that $\partial H_{t} /\left.\partial u_{1 t}\right|_{u_{1 t}=1} \geq 0$. 
(39) is equivalent to

$$
\sum_{i=0}^{1}\left(\frac{1}{\rho_{i}}-\frac{1}{B_{1}}\right) \pi_{i} e^{-\rho_{i} 0} \leq \sum_{i=0}^{1}\left(\frac{1}{\rho_{i}}-\frac{1}{B_{0}}\right) \pi_{i} e^{-\rho_{i} T_{0}}
$$

which implies

$$
\sum_{i=0}^{1}\left(\frac{1}{\rho_{i}}-\frac{1}{B_{1}}\right) \pi_{i} e^{-\rho_{i} t} \leq \sum_{i=0}^{1}\left(\frac{1}{\rho_{i}}-\frac{1}{B_{0}}\right) \pi_{i} e^{-\rho_{i} T_{0}}
$$

for any $t \in\left[0, T_{0}\right]$, because the LHS of (91), under Assumption 5, is a decreasing function of $t$. By definition of $T_{0}$,

$$
\sum_{i=0}^{1}\left(\frac{1}{\rho_{i}}-\frac{1}{B_{0}}\right) \pi_{i} e^{-\rho_{i} T_{0}}=\frac{1}{B_{0}} \frac{H_{00}}{H_{10}} \sum_{i=0}^{1} \pi_{i} e^{\left(B_{0}-\rho_{i}\right) T_{0}}
$$

Inequality (91) becomes

$$
\sum_{i=0}^{1}\left(\frac{1}{\rho_{i}}-\frac{1}{B_{1}}\right) \pi_{i} e^{-\rho_{i} t} \leq \frac{1}{B_{0}} \frac{H_{00}}{H_{10}} \sum_{i=0}^{1} \pi_{i} e^{\left(B_{0}-\rho_{i}\right) T_{0}}=\sum_{i=0}^{1}\left(\frac{1}{\rho_{i}}-\frac{1}{B_{0}}\right) \pi_{i} e^{-\rho_{i} T_{0}}
$$

for any $t \in\left[0, T_{0}\right]$. (89) and (87) imply

$$
\sum_{i=0}^{1}\left(\frac{1}{\rho_{i}}-\frac{1}{B_{0}}\right) \pi_{i} e^{-\rho_{i} T_{0}}=\sum_{i=0}^{1} \frac{\pi_{i}}{\rho_{i}} e^{-\rho_{i} t}-\lambda_{1 t} h_{10}
$$

Therefore, (92) becomes

$$
B_{1} \lambda_{1 t} \leq \frac{\sum_{i=0}^{1} \pi_{i} e^{-\rho_{i} t}}{h_{10}}=\pi_{1} \frac{\sum_{i=0}^{1} \pi_{i} e^{-\rho_{i} t}}{\sum_{i=0}^{1} \pi_{i} h_{i t} u_{i t}}
$$

(because $u_{0 t}=0$ for $0 \leq t \leq T_{0}$ and $u_{1 t}=1$ for any $t$ ), that is, according to (82), $\partial H_{t} /\left.\partial u_{1 t}\right|_{u_{1 t}=1} \geq 0$. This means that $u_{1 t}=1$ maximizes the Hamiltonian.

(2) Focus now on the case $B_{1}>B_{1}^{*}$.

Lemma 9 allows us to focus on a candidate solution which is corner solution for one agent. We prove the second subcase by considering a potential 
solution such that

$$
\begin{aligned}
u_{0 t} & =0 \text { for } 0 \leq t \leq T_{2} \\
0 & <u_{0 t}<1 \text { for } t>T_{2} \\
0 & <u_{1 t}<1 \text { for } 0 \leq t<T_{1} \\
u_{1 t} & =1 \text { for } t \geq T_{1}
\end{aligned}
$$

with $T_{1}<T_{2}$, by deriving its explicit trajectory and checking that this trajectory satisfies the Pontryagin's Maximum Principle.

Applying the arguments of point (2.1) to determine $T_{0}$ (namely, the transversality condition), we find $T_{2}$ as solution of

$$
\begin{aligned}
\frac{B_{0}}{\rho\left(T_{2}\right)} & =1+\frac{H_{0 T_{2}}}{H_{1 T_{2}}} \\
& =1+\frac{H_{00}}{H_{10}} \frac{e^{B_{0} T_{2}}}{e^{B_{1} T_{1}}\left[1+\frac{1}{\lambda_{10} h_{10}} \sum_{i=0}^{1} \frac{\pi_{i}}{\rho_{i}}\left(e^{-\rho_{i} T_{1}}-1\right)\right]}
\end{aligned}
$$

that is of $(36)$.

For $0 \leq t \leq T_{1}$ we have

$$
\left.\frac{\partial H_{t}}{\partial u_{1 t}}\right|_{0<u_{1 t}<1}=\pi_{1} h_{1 t} \frac{\sum_{i=0}^{1} \pi_{i} e^{-\rho_{i} t}}{\sum_{i=0}^{1} \pi_{i} h_{i t} u_{i t}}-B_{1} \lambda_{1 t} h_{1 t}=0
$$

that is

$$
B_{1} \lambda_{1 t}=\frac{\sum_{i=0}^{1} \pi_{i} e^{-\rho_{i} t}}{h_{1 t} u_{1 t}}
$$

since $u_{0 t}=0$ and $0<u_{1 t}<1$ (because $T_{1}<T_{2}$ ).

Moreover, using (93), we find

$$
\dot{\lambda}_{1 t}=-\frac{\partial H_{t}}{\partial h_{1 t}}=-\pi_{1} u_{1 t} \frac{\sum_{i=0}^{1} \pi_{i} e^{-\rho_{i} t}}{\sum_{i=0}^{1} \pi_{i} h_{i t} u_{i t}}-B_{1} \lambda_{1 t}\left(1-u_{1 t}\right)=-B_{1} \lambda_{1 t}
$$

Therefore, for $0 \leq t \leq T_{1}, \lambda_{1 t}=\lambda_{10} e^{-B_{1} t}$ and, from (93),

$$
h_{1 t} u_{1 t}=\frac{\sum_{i=0}^{1} \pi_{i} e^{-\rho_{i} t}}{B_{1} \lambda_{1 t}}=\frac{1}{B_{1} \lambda_{10}} \sum_{i=0}^{1} \pi_{i} e^{\left(B_{1}-\rho_{i}\right) t}
$$


The law of motion $\dot{h}_{1 t}=B_{1}\left(1-u_{1 t}\right) h_{1 t}$ and (94) imply

$$
\dot{h}_{1 t}=B_{1} h_{1 t}-\frac{1}{\lambda_{10}} \sum_{i=0}^{1} \pi_{i} e^{\left(B_{1}-\rho_{i}\right) t}
$$

We define

$$
z_{t} \equiv h_{1 t}-\frac{1}{\lambda_{10}} \sum_{i=0}^{1} \frac{\pi_{i}}{\rho_{i}} e^{\left(B_{1}-\rho_{i}\right) t}
$$

Using (95), it is easy to verify that $\dot{z}_{t}=B_{1} z_{t}$. Thus, $z_{t}=z_{1} e^{B_{1} t}$. From (96), we obtain

$$
h_{1 t}=z_{1} e^{B_{1} t}+\frac{1}{\lambda_{10}} \sum_{i=0}^{1} \frac{\pi_{i}}{\rho_{i}} e^{\left(B_{1}-\rho_{i}\right) t} \text { with } z_{1}=h_{10}-\frac{1}{\lambda_{10}} \sum_{i=0}^{1} \frac{\pi_{i}}{\rho_{i}}
$$

(95) and (97) imply $\dot{h}_{1 t}=e^{B_{1} t} \phi(t)$ where

$$
\phi(t) \equiv B_{1} h_{10}-\frac{1}{\lambda_{10}} \sum_{i=0}^{1} \pi_{i}\left[\frac{B_{1}}{\rho_{i}}+\left(1-\frac{B_{1}}{\rho_{i}}\right) e^{-\rho_{i} t}\right]
$$

Assume that

$$
\frac{1}{B_{1} h_{10}}<\lambda_{10}<\frac{1}{h_{10}} \sum_{i=0}^{1} \frac{\pi_{i}}{\rho_{i}}
$$

Therefore,

$$
\phi(0)=B_{1} h_{10}-\frac{1}{\lambda_{10}}>0>B_{1} h_{10}-\frac{B_{1}}{\lambda_{10}} \sum_{i=0}^{1} \frac{\pi_{i}}{\rho_{i}}=\phi(\infty)
$$

Thus, $\phi(t)=0$ (that is $\dot{h}_{1 t}=0$ ) has solution. Let $T_{1}>0$ be the smallest solution. This solution depends on $\lambda_{10} . \phi\left(T_{1}\right)=0$ is equivalent to (37). Function $T_{1}\left(\lambda_{10}\right)$ behaves as follows.

$$
\begin{aligned}
& \lambda_{10} \rightarrow \frac{1}{B_{1} h_{10}} \Leftrightarrow T_{1}\left(\lambda_{10}\right) \rightarrow 0 \\
& \lambda_{10} \rightarrow \frac{1}{h_{10}} \sum_{i=0}^{1} \frac{\pi_{i}}{\rho_{i}} \Leftrightarrow T_{1}\left(\lambda_{10}\right) \rightarrow \infty
\end{aligned}
$$


There exists a constant $C$ such that $h_{1 t}<C e^{B_{1} t}$ for any $t \geq 0$. Hence for $T_{1}$ big enough we have

$$
\frac{h_{0 T_{1}}}{h_{1 T_{1}}}>\frac{e^{B_{0} T_{1}}}{C e^{B_{1} T_{1}}}=\frac{e^{\left(B_{0}-B_{1}\right) T_{1}}}{C}>\frac{\pi_{1}}{\pi_{0}}\left[\frac{B_{0}}{\rho\left(T_{1}\right)}-1\right]
$$

or, equivalently, $B_{0} / \rho\left(T_{1}\right)<1+H_{0 T_{1}} / H_{1 T_{1}}$. Since $B_{0} / \rho(0)>1+H_{00} / H_{10}$, there exists $\hat{\lambda}$ such that for $T_{1}=T_{1}(\hat{\lambda})$ :

$$
\frac{B_{0}}{\rho\left(T_{1}\right)}=1+\frac{H_{0 T_{1}}}{H_{1 T_{1}}}
$$

We observe that $T_{1}\left(\lambda_{10}\right)<T_{2}\left(\lambda_{10}\right)$ for $1 /\left(B_{1} h_{10}\right)<\lambda_{10}<\hat{\lambda}$. Differentiating $\phi(t)=0$ with respect to $t$ and $\lambda_{10}$, we find at $t=T_{2}$ :

$$
T_{1}^{\prime}\left(\lambda_{10}\right)=\frac{B_{1} h_{10} / \sum_{j=0}^{1} \pi_{j} e^{-\rho_{j} T_{1}}}{B_{1}-\sum_{i=0}^{1} \rho_{i} \frac{\pi_{i} e^{-\rho_{i} T_{1}}}{\sum_{j=0}^{1} \pi_{j} e^{-\rho_{j} T_{1}}}}>0
$$

Moreover, $T_{1}(\hat{\lambda})=T_{2}(\hat{\lambda})$.

Therefore,

$$
\dot{\lambda}_{1 t}=-\frac{\partial H_{t}}{\partial h_{1 t}}=-\pi_{1} u_{1 t} \frac{\sum_{i=0}^{1} \pi_{i} e^{-\rho_{i} t}}{\sum_{i=0}^{1} \pi_{i} h_{i t} u_{i t}}-B_{1} \lambda_{1 t}\left(1-u_{1 t}\right)=-\frac{\sum_{i=0}^{1} \pi_{i} e^{-\rho_{i} t}}{h_{1 T_{1}}}
$$

At $t=T_{1}$, we have $\partial H_{t} / \partial u_{1 t}=0$, that is

$$
\lambda_{1 T_{1}}=\frac{\sum_{i=0}^{1} \pi_{i} e^{-\rho_{i} T_{1}}}{B_{1} h_{1 T_{1}}}
$$

Integrating (98) from $T_{1}$ to $t \leq T_{2}$, we get

$$
\lambda_{1 t}=\lambda_{1 T_{1}}+\frac{1}{h_{1 T_{1}}} \sum_{i=0}^{1} \frac{\pi_{i}}{\rho_{i}}\left(e^{-\rho_{i} t}-e^{-\rho_{i} T_{1}}\right)
$$

Replacing (99), we obtain

$$
\lambda_{1 t}=\frac{1}{h_{1 T_{1}}} \sum_{i=0}^{1} \frac{\pi_{i}}{\rho_{i}}\left[e^{-\rho_{i} t}+\left(\frac{\rho_{i}}{B_{1}}-1\right) e^{-\rho_{i} T_{1}}\right] \text { for } T_{1} \leq t \leq T_{2}
$$


and, in particular, (51).

At time $T_{2}$, the optimal solution satisfies $\partial H_{t} / \partial u_{0 t}=0$ or, equivalently,

$$
B_{0} \lambda_{0 t}=\pi_{0} \frac{\sum_{i=0}^{1} \pi_{i} e^{-\rho_{i} t}}{\sum_{i=0}^{1} \pi_{i} h_{i t} u_{i t}}
$$

that is

$$
\lambda_{0 T_{2}}=\frac{\pi_{0}}{B_{0} H_{1 T_{1}}} \sum_{i=0}^{1} \pi_{i} e^{-\rho_{i} T_{2}}
$$

Using (101), we find

$$
\dot{\lambda}_{1 t}=-\frac{\partial H_{t}}{\partial h_{1 t}}=-\pi_{1} u_{1 t} \frac{\sum_{i=0}^{1} \pi_{i} e^{-\rho_{i} t}}{\sum_{i=0}^{1} \pi_{i} h_{i t} u_{i t}}-B_{1} \lambda_{1 t}\left(1-u_{1 t}\right)=-\frac{\pi_{1}}{\pi_{0}} B_{0} \lambda_{0 t}=\frac{\pi_{1}}{\pi_{0}} \dot{\lambda}_{0 t}
$$

Integrating (98) from $T_{1}$ to $t \leq T_{2}$, we get

$$
\lambda_{1 t}=\frac{\pi_{1}}{\pi_{0}}\left(\lambda_{0 t}-\lambda_{0 T_{1}}\right)+\lambda_{1 T_{1}}=\frac{\pi_{1}}{\pi_{0}} \lambda_{0 T_{1}} e^{-B_{0}\left(t-T_{1}\right)}+\lambda_{1 T_{1}}-\frac{\pi_{1}}{\pi_{0}} \lambda_{0 T_{1}}
$$

A candidate solution satisfies the transversality condition, that is

$\lim _{t \rightarrow \infty}\left(\lambda_{1 t} h_{1 t}\right)=\lim _{t \rightarrow \infty}\left[\frac{\pi_{1}}{\pi_{0}} \lambda_{0 T_{1}} e^{-B_{0}\left(t-T_{1}\right)} h_{1 t}\right]+\lim _{t \rightarrow \infty}\left[\left(\lambda_{1 T_{1}}-\frac{\pi_{1}}{\pi_{0}} \lambda_{0 T_{1}}\right) h_{1 t}\right]=0$

which holds only if $\lambda_{1 T_{1}}-\lambda_{0 T_{1}} \pi_{1} / \pi_{0}=0$. This implies $\lambda_{1 t}=\lambda_{0 t} \pi_{1} / \pi_{0}$. In particular, at the point $T_{2}>T_{1}$, we have $\lambda_{1 T_{2}}=\lambda_{0 T_{2}} \pi_{1} / \pi_{0}$ and, according to $(102)$,

$$
\lambda_{1 T_{2}}=\frac{\pi_{1}}{\pi_{0}} \lambda_{0 T_{2}}=\frac{1}{B_{0} h_{1 T_{1}}} \sum_{i=0}^{1} \pi_{i} e^{-\rho_{i} T_{2}}
$$

Using (51), we obtain (38). To conclude, we must prove that there exists $\lambda_{10}$ with

$$
\frac{1}{h_{10}} \sum_{i=0}^{1} \frac{\pi_{i}}{\rho_{i}}<\lambda_{10}<\hat{\lambda}
$$

such that the equation (38) is verified. Indeed, for

$$
\lambda_{10}=\frac{1}{h_{10}} \sum_{i=0}^{1} \frac{\pi_{i}}{\rho_{i}}
$$


we have $T_{1}\left(\lambda_{10}\right)=0$ and $T_{2}\left(\lambda_{10}\right)=T_{0}$ and, so,

$$
f\left(\lambda_{10}\right) \equiv \frac{\frac{1}{\rho\left(T_{0}\right)}-\frac{1}{B_{0}}}{\frac{1}{\rho(0)}-\frac{1}{B_{1}}}-\frac{1}{\sum_{i=0}^{1} \pi_{i} e^{-\rho_{i} T_{0}}}<0
$$

because of (??). For $\lambda=\hat{\lambda}$, we have $T_{1}\left(\lambda_{10}\right)=T_{2}\left(\lambda_{10}\right)$ and, so,

$$
f\left(\lambda_{10}\right) \equiv \frac{\frac{1}{\rho\left(T_{1}\left(\lambda_{10}\right)\right)}-\frac{1}{B_{0}}}{\frac{1}{\rho\left(T_{1}\left(\lambda_{10}\right)\right)}-\frac{1}{B_{1}}}-1>0
$$

since $B_{0}>B_{1} . f$ is a continuous function. Then, there exists $\lambda_{10}$ such that $f\left(\lambda_{10}\right)=0$, that is $(38)$ is satisfied.

Finally, we can construct the explicit solution with $\lambda_{10}$ solution of (38).

We have $\lambda_{0 t}=\lambda_{00} e^{-B_{0} t}$ for any $t$ with $\lambda_{00}=\lambda_{1 T_{2}} e^{B_{0} T_{2}} \pi_{0} / \pi_{1}$. Moreover, $h_{0 t}=h_{00} e^{B_{0} t}$ for $0 \leq t \leq T_{2}$, while for $t \geq T_{2}$, we obtain (47) similarly to (41). We have also $u_{0 t}=0$ for $0 \leq t \leq T_{2}$, while, for $t \geq T_{2}$, we obtain (48) similarly to (43).

For $0 \leq t \leq T_{1}$, from (94) and (97), we obtain (50). We observe that $h_{1 t}=h_{1 T_{1}}$ for $t \geq T_{1}$.

For $0 \leq t \leq T_{1}$, it is easy to see that $\lambda_{1 t}=\lambda_{10} e^{-B_{0} t}$, while, for $T_{1} \leq t \leq T_{2}$, $\lambda_{1 t}$ is given by (100). For $t \geq T_{2}$, we find $\lambda_{1 t}=\lambda_{1 T_{2}} e^{-B_{0}\left(t-T_{2}\right)}$.

Proof of Proposition 15. Under the assumption $h_{0}>h_{1}$, the Gini index of wealth is given by:

$$
g_{h t}=\frac{\frac{\pi_{0} h_{0 t}+\pi_{1} h_{1 t}}{2}-\left(\frac{\pi_{1} \pi_{1} h_{1 t}}{2}+\pi_{0} \pi_{1} h_{1 t}+\frac{\pi_{0} \pi_{0} h_{0 t}}{2}\right)}{\frac{\pi_{0} h_{0 t}+\pi_{1} h_{1 t}}{2}}
$$

that is by

$$
g_{h t}=\pi_{1}-\frac{\pi_{1} h_{1 t}}{\pi_{0} h_{0 t}+\pi_{1} h_{1 t}}=\pi_{1}-\frac{H_{1 t}}{H_{0 t}+H_{1 t}}
$$

or, equivalently, by (55).

Proof of Proposition 16. According to (25), $\rho_{0}<\rho_{1}$ implies $c_{0 t}>c_{1 t}$. Thus, the Gini index of consumption is given by

$$
g_{c t}=\pi_{1}-\frac{\pi_{1} c_{1 t}}{\pi_{0} c_{0 t}+\pi_{1} c_{1 t}}
$$

Replacing (25) in (103) we get (56). 
Proof of Proposition 17. Focus on Proposition 10. According to (25), we obtain the aggregate consumption:

$$
c_{t}=\sum_{i=0}^{1} \pi_{i} A e^{-\rho_{i} t} \frac{\sum_{j=0}^{1} \pi_{j} h_{j t} u_{j t}}{\sum_{j=0}^{1} \pi_{j} e^{-\rho_{j} t}}=A\left(\pi_{0} h_{0 t} u_{0 t}+\pi_{1} h_{1 t} u_{1 t}\right)
$$

and, so, replacing expressions (30) to (33), we find (57). Computing the time derivative of (57), we obtain the aggregate consumption growth rate (58).

Proof of Proposition 18. From equation (56), we find

$$
e^{\left(B_{0}-\rho_{i}\right) t}=\left(\frac{\pi_{1}}{\pi_{0}} \frac{\pi_{0}+g_{c t}}{\pi_{1}-g_{c t}}\right)^{\frac{B_{0}-\rho_{i}}{\rho_{1}-\rho_{0}}}
$$

Replacing (104) in (58) we find (59). Finally, it is easy to show that $\gamma_{c}$ is an increasing function of $g_{c}$.

\section{References}

[1] Abramovitz M. and P.A. David (2000). American macroeconomic growth in the era of knowledge-based progress: the long-run perspective. In S.L. Engerman and R.E. Gallman editors, The Cambridge Economic History of the United States, Cambridge University Press, New York.

[2] Alesina A. and D. Rodrik (1994). Distributive politics and economic growth. Quarterly Journal of Economics 109, 465-490.

[3] Atkinson A.B. (1999). The distribution of income in the UK and OECD countries in the twentieth century. Oxford Review of Economic Policy, $15,56-75$.

[4] Banerjee A. and A.F. Newman (1993). Occupational choice and the process of development. Journal of Political Economy 101, 274-298.

[5] Barro R.J. (2000). Inequality and growth in a panel of countries. Journal of Economic Growth 5, 5-32.

[6] Becker G.S. (1962). Investment in human capital: a theoretical analysis. Journal of Political Economy 70, 9-49. 
[7] Becker G.S. (1964). Human Capital: A Theoretical and Empirical Analysis, with Special Reference to Education. National Bureau of Economic Research, New York.

[8] Becker G.S. and N. Tomes (1979). An equilibrium theory of the distribution of income and intergenerational mobility. Journal of Political Economy 87, 1153-1189.

[9] Becker R.A. (2006). Equilibrium dynamics with many agents. In R.-A. Dana, C. Le Van, T. Mitra, and K. Nishimura editors, Handbook of Optimal Growth 1. Springer-Verlag.

[10] Bourguignon F. (1981). Pareto-superiority of unegalitarian equilibria in Stiglitz' model of wealth distribution with convex savings function. Econometrica 49, 1469-1475.

[11] Campbell J. (1999). Asset prices, consumption and the business cycle. In J.B. Taylor and M. Woodford editors, Handbook of Macroeconomics, North-Holland, Amsterdam, 1231-1303.

[12] Dohmen T., B. Enke, A. Falk, D. Huffman and U. Sunde (2015). Patience and the wealth of nations. Mimeo.

[13] Eckstein Z. and I. Zilcha (1994). The effects of compulsory schooling on growth, income distribution and welfare. Journal of Public Economics 54, 339-359.

[14] Eicher T.S. and C. García-Peñalosa (2001). Inequality and growth: the dual role of human capital in development. Journal of Development Economics, 66, 173-197.

[15] Falk A., A. Becker, T. Dohmen, B. Enke, U. Sunde and D. Huffman (2015). The nature of human preferences: global evidence. CESIFO Area Conference on Behavioral Economics, 23-24 October.

[16] Forbes K. (2000). A reassessment of the relationship between inequality and growth. American Economic Review 90, 869-887.

[17] Frank M. (2009). Inequality and growth in the United States: evidence from a new state-panel of income inequality measures. Economic Inquiry $47,55-68$. 
[18] Galor O. and J. Zeira (1993). Income distribution and macroeconomics. Review of Economic Studies 60, 35-52.

[19] Galor O. and O. Moav (2004). From physical to human capital accumulation: inequality and the process of development. Review of Economic Studies 71, 1001-1126.

[20] Glomm G. and B. Ravikumar (1992). Public versus private investment in human capital: endogenous growth and income inequality. Journal of Political Economy 100, 818-834.

[21] Goldin C. and L.F. Katz (1999). The returns to skill across the twentieth century United States. Working Paper NBER 7126, Cambridge, MA.

[22] Goldin C. and L.F. Katz (2001). The legacy of U.S. educational leadership: notes on distribution and economic growth in the 20th century." American Economic Review, Papers and Proceedings 91, 18-23.

[23] Goldin C. and L.F. Katz (2008). The Race between Education and Technology. Harvard University Press, Cambridge, MA.

[24] Heal, G. and Millner, A. (2015). Collective Intertemporal Choice: Time Consistency vs. Time Invariance. Working Paper 247, Centre for Climate Change Economics and Policy.

[25] Hübner M. and G. Vannoorenberghe (2015). Patience and long-run growth, Economics Letters 137, 163-167.

[26] Jones, B.F. (2014). The human capital stock: a generalized approach. American Economic Review 104, 3752-3777.

[27] Lucas R.E. (1988). On the mechanics of economic development. Journal of Monetary Economics 22, 3-42.

[28] Lucas R.E. (2015). Reflections of new growth theory, human capital and growth. American Economic Review, Papers and Proceedings 105, 85-88.

[29] Manuelli R.E. and A. Seshadri (2014). Human capital and the wealth of nations. American Economic Review 104, 2736-2762.

[30] Mincer J. (1958). Investment in human capital and personal income distribution. Journal of Political Economy 66, 281-302. 
[31] Mincer J. (1962). On-the-job training: costs, returns, and some implications. Journal of Political Economy 70, 50-79.

[32] Partridge M.D. (1997). Is inequality harmful for growth? Comment. American Economic Review 87, 1019-1032.

[33] Perottti R. (1996). Growth, income distribution and democracy: what the data say. Journal of Economic Growth 1, 149-187.

[34] Persson T. and G. Tabellini (1994). Is inequality harmful for growth? Theory and evidence. American Economic Review 84, 600-621.

[35] Rebelo S. (1991). Long-run policy analysis and long-run growth. Quarterly Journal of Economics 107, 255-284.

[36] Schultz T.W. (1961). Investment in human capital. American Economic Review 51, 1-17.

[37] Schultz T.W. (1962). Reflections on investment in man. Journal of Political Economy 70, 1-8.

[38] Saint-Paul G. and T. Verdier (1993). Education, democracy and growth. Journal of Development Economics 42, 399-407.

[39] Stiglitz J.E. (1969). Distribution of income and wealth among individuals. Econometrica 37, 382-397.

[40] Turnovsky S.J. (2011). The accumulation of human capital and income inequality in a two-sector economy. Journal of Human Capital 5, 418452.

[41] Turnovsky S.J. and A. Mitra (2013). The interaction between human and physical capital accumulation and the growth-inequality trade-off. Journal of Human Capital 7, 26-75.

[42] Uzawa H. (1965). Optimum technical change in an aggregative model of economic growth. International Economic Review 6, 18-31.

[43] Viaene J.-M. and I. Zilcha (2003). Human capital formation, income inequality, and growth. In T.S. Eicher and S.J. Turnovsky editors. Inequality and Growth: Theory and Policy Implications. MIT Press, Cambridge, MA. 
[44] Zuber, S. 2011. "The aggregation of preferences: Can we ignore the past?" Theory and Decision 70 (1): 367-84. 